\title{
Propagation of rays in 2D and 3D waveguides: a stability analysis with Lyapunov and Reversibility fast indicators
}

\author{
G. Gradoni, ${ }^{1, a)}$ F. Panichi, ${ }^{2, b)}$ and G. Turchetti ${ }^{3,(\mathrm{c})}$ \\ ${ }^{1)}$ School of Mathematical Sciences and Department of Electrical and Electronic Engineering, University of Nottingham, \\ United Kingdom ${ }^{\text {d) }}$ \\ 2) Department of Physics and Astronomy, University of Bologna, Italy \\ 3) Department of Physics and Astronomy, University of Bologna, Italy. INDAM National Group of Mathematical Physics, \\ Italy \\ (Dated: 13 January 2021)
}

Propagation of rays in 2D and 3D corrugated waveguides is performed in the general framework of stability indicators. The analysis of stability is based on the Lyapunov and Reversibility error. It is found that the error growth follows a power law for regular orbits and an exponential law for chaotic orbits. A relation with the Shannon channel capacity is devised and an approximate scaling law found for the capacity increase with the corrugation depth.

We investigate the propagation of a ray in a 2D wave guide whose boundaries are two parallel horizontal lines, with a periodic corrugation on the upper line. The reflection point abscissa on the lower line and the ray horizontal velocity component after reflection are the phase space coordinates and the map connecting two consecutive reflections is symplectic. The dynamic behaviour is illustrated by the phase portraits which show that the regions of chaotic motion increase with the corrugation amplitude. For a 3D wave-guide the 4D map connecting two consecutive reflections on the lower plane is symplectic, but its orbit cannot be examined by looking at the intersections with a 2D phase plane, since a continuous interpolation of the orbits is not available. In this case the fast dynamic indicators allow to perform a stability analysis. For each point of a grid in a 2D phase plane one computes the orbit for a chosen number of iterations and the corresponding value of the fast indicator, which is conveniently visualized using a color plot. After the fast Lyapunov indicator, many other indicators have been introduced. Our analysis is based on the Lyapunov error (LE), due to a small random initial displacement and the reversibility error occurring when the orbit is reversed in presence of a small additive noise (RE) or round off (REM). For integrable maps the growth of LE and RE follows a power law and for quasi integrable maps the same growth occurs close to a stable fixed point. More generally the error growth follows a power law for regular orbits and an exponential law for chaotic orbits. There is numerical evidence that REM grows as RE though with large fluctuations. The channel capacity is related to $L E$ and the dependence of its phase space average on the corrugation amplitude is considered. These indicators confirm their reliability for the stability analysis of the ray propagation in a $2 D$ and $3 D$ wave guide, providing a measure of the sensitivity of the orbits to initial conditions, noise and round off.

\footnotetext{
a)Electronic mail: gabriele.gradoni@nottingham.ac.uk.

b)Electronic mail: federico.panichi@ studio.unibo.it.

c) Electronic mail: giorgio.turchetti@unibo.it.

d) Also at Maxwell Centre, University of Cambridge, United Kingdom.
}

\section{INTRODUCTION}

The equivalence between geometrical optics and mechanics was established in a variational form by the principles of Fermat and Maupertuis. If a ray propagates in a uniform medium with a reflecting boundary, then the trajectory is the same as a particle freely moving and elastically colliding with the same boundary. As consequence a wave-guide and a billiard are equivalent optical and mechanical systems ${ }^{1}$. Since the velocity of the particle does not change, we can assume it has unit modulus, just as the ray velocity normalized to the speed of light. The trajectory is determined by the collision points and the velocity direction after the collision. The billiards with polygonal or smooth convex closed boundaries have been intensively investigated and the mathematical literature is very rich, see $e^{2-[5]}$.

We first consider the 2D wave-guide whose boundary is a straight line and a corrugated parallel line. The trajectory is a polygonal line specified by the abscissa $x$ of the reflection points on the straight line and the parallel component $v_{x}$ of the velocity after reflection. The Fermat principle establishes that, given two points $x_{1}, x_{2}$ on the lower straight line, the ray, colliding once with the corrugated line, follows the path of minimal length and the collision is just a reflection. In addition the ray minimal path length $h\left(x_{1}, x_{2}\right)$ is the generating function of the area preserving map $M$ connecting two subsequent reflections on the straight line. A similar procedure allows to obtain a symplectic map for the ray propagation in a $3 \mathrm{D}$ waveguide made of an horizontal plane and an upper parallel plane with a periodic corrugation. If the medium between the waveguide boundaries if not uniform the piecewise linear path joining two consecutive reflection points on the lower line or plane is replaced by the geodesic with respect to the metric $n d s$, where $n$ is the refraction index.

The transition from ordered to chaotic motion in 2D billiards and waveguides has been considered ${ }^{6 / 8}$; the transport and diffusion properties have been extensively analysed $9+15$. The stability properties of the map depend on the corrugation amplitude. For the $2 \mathrm{D}$ wave guide the phase portrait 
of the corresponding 2D map allows to detect the regions of regular and chaotic motion. Finite time indicators such as the Fast Lyapunov Indicator (FLI) $\stackrel{1617]}{17}$ have been first proposed to analyze the orbital stability. Other short term indicators of variational nature have been introduced: the Alignment Indices (SALI) ${ }^{18}$, the Orthogonal Fast Lyapunov Indicator (OFLI) ${ }^{20}$, the Mean Exponential Growth of Nearby Orbits (MEGNO) ${ }^{\sqrt{2122}}$, which is an excellent filter of the oscillations of FLI, the relative Lyapunov indicator (RLI) ${ }^{23}$, and the Generalized Lyapunov indicators (GALI), whose asymptotic behaviour is related the all the Lyapunov exponents ${ }^{24 / 25}$. An indicator based on the distribution of the stretching numbers (SSN) was also proposed 26127. An extensive numerical comparison of previous indicators for symplectic maps was presented 28129. Spectral indicators based on the Fourier analysis have also been proposed and extensively used ${ }^{31}$. A geometric chaos indicator based on the Riemann curvature of the constant energy manifold $32\left[33\right.$ and a $0-1$ test of chaos ${ }^{34}$ have also been proposed. More recently the Lyapunov and reversibility errors have been introduced ${ }^{35136}$ to measure the sensitivity of the orbits to a small random initial displacement and to a small additive noise along the orbit. The Lyapunov error (LE) and the reversibility error are simply related ${ }^{37}$ so that the computation of RE does not require expensive MonteCarlo procedures. The relevant difference of LE withe respect to previous variational indicators such as FLI, is that LE does not depend on the initial deviation vectors. A full set of Lyapunov error indicators (LEI), whose asyptotic behaviour is related to all the Lyapunov exponents, just as GALI, and which independent from the initial deviation vectors, has been introduced jointly with reversibility error indicators (REI) 38 . The last REI corresponds to the reversibility entropy, and its asymptotic behaviour is governed by the sum of positive Lyapunov exponents, as the upper-bound to the KolmogorovSinali entropy 30 .

The LE and RE grow following a power law, for the regular orbits of quasi integrable symplectic maps as the previous variational indicators, exponentially for chaotic orbits. The reversibility error due round off (REM) was first introduced in 39 and its features were examined in ${ }^{40}$. For previous works on the round effect in the computation of orbits for Hamiltonian systems see ${ }^{41}$. Previous numerical investigations of Hamiltonian systems and symplectic maps confirm that LE grows linearly with oscillations, (due to the loss of rotational symmetry when the coordinates are not normal) for regular orbits, whereas the growth of RE is almost oscillations free. Neglecting its large fluctuations REM is comparable with RE, even though no rigorous proof is available.

For the 3D waveguide no direct inspection of the orbits is possible, since the section of the orbits of the symplectic 4D map with a $2 \mathrm{D}$ plane would require a continuous interpolation, available only when an interpolating Hamiltonian is known. Normal forms provide the interpolating Hamiltonian for quasi integrable symplectic maps, but their recursive computation is possible just for polynomial maps, and in addition the interpolation is not exact due to the presence of a non integrable remainder. The variational indicators, computed for the orbits issued from the points of a regular grid in a $2 \mathrm{D}$ phase plane for the 4D map, and visualized with a colour plot, allow to determine the stability properties just as for the 2D map. We have analyzed only LE and RE for a limited number of iteration. Indeed as stated Froeschlé et al. the variational indicators as FLI computed for short times exhibit some dependency on the initial conditions of the deviation vectors. Since LE, RE and REM do not depend on the initial deviation vectors our choice is justified. A careful investigation of the sticky chaos, which requires longer orbits, and more extensive numerical exploration of the reflection maps based on the full set of invariant indicators LEI and REI and a comparison with the standard variational indicators, will be the object of a future work.

Only a few hundred iterations of the map are required to obtain a reliable stability picture, unless one is interested in the details of a small region. To obtain the analytic form of the tangent map is rather cumbersome for the $3 \mathrm{D}$ waveguide case. The shadow orbit method provides a simple, though less accurate, alternative which consists in evaluating the orbits for initial conditions with small displacements along an orthogonal basis, which amounts to replace the partial derivatives with finite differences. For any initial condition, 4 additional evaluations of the orbit are required ( 2 for the 2D map) in order to obtain $\mathrm{LE}$ and $\mathrm{RE}$, whereas just 1 is required to compute REM.

We have analyzed a model of 2D waveguide for different values of the corrugation amplitude, showing that the LE, RE, REM provide comparable results, which describe the orbits sensitivity to a small initial random displacement, to a noise along the orbit and to round off. For a model of 3D waveguide the same error plots, for initial conditions on 2D phase planes, exhibit a similar behaviour, though the structure is richer with respect to the $2 \mathrm{D}$ waveguide, due to the presence of the Arnold web of resonances The effectiveness of the proposed method, already experienced in celestial mechanic $\$ 35 / 36$ and beam dynamics ${ }^{42}$ models, is confirmed in these examples of $2 \mathrm{D}$ and $3 \mathrm{D}$ waveguides.

The paper is organised as follows. In section 1 we present the variational derivation of the reflections map for a $2 \mathrm{D}$ waveguide. In section 3 the extension to the $3 \mathrm{D}$ waveguide is outlined. In section 4 our dynamical indicators are defined and their basic properties are illustrated. In section 5 and 6 the numerical results of these indicators show how the instability regions grow when the corrugation amplitude increases. In section 7 the link between our indicators and the channel capacity is established and it is shown how its phase space average increases with the corrugation amplitude. Conclusions and perspectives are presented in section 8 .

\section{THE 2D WAVEGUIDE}

Given two parallel reflecting lines $z=0$ and $z=1$ in the $(x, z)$ plane, the light ray direction after each reflection on the lower line is the same being specified by the unit vector $\mathbf{v}=\left(x_{x}, v_{z}=\sqrt{1-v_{x}^{2}}\right)^{T}$, where $T$ denotes the transpose of a matrix or a vector. The time $\tau$ between two reflection on the upper and lower line is given by $\tau v_{z}=1$. We choose as phase space coordinates $\left(x, v_{x}\right)$ so that the sequence of reflections 
$\left(x_{n}, v_{x n}\right)$ on the lower line is given by

$$
\begin{aligned}
& v_{x n+1}=v_{x n} \\
& x_{n+1}=x_{n}+2 \tau v_{x n}=x_{n}+\frac{2 v_{x n}}{\sqrt{1-v_{x n}^{2}}}
\end{aligned}
$$

This map is integrable and area preserving. If we let $\mathbf{v}=$ $(\cos \theta, \sin \theta)^{T}$ where $0 \leq \theta \leq \pi$ the map becomes

$$
\theta_{n+1}=\theta_{n} \quad x_{n+1}=x_{n}+2 \cot \theta_{n}
$$

and preserves the measure $d \mu=\sin \theta d x d \theta$. If our phase space is a cylinder $\mathbb{T}([-\pi, \pi])] \times[-1,1]$ rather than the infinite strip $\mathbb{R} \times[-1,1]$, then $x$ is an angle variable and $v_{x}$ an action variable.

The frequency $\Omega=2 v_{x}\left(1-v_{x}^{2}\right)^{-1 / 2}$ diverges for $v_{x} \rightarrow \pm 1$ and the interpolating Hamiltonian is $H=-2 \sqrt{1-v_{x}^{2}}$. Since also $\Omega^{\prime}$ diverges when $v_{x} \rightarrow \pm 1$, any perturbation renders the map chaotic as for an integrable near the the separatrix where $\Omega$ vanishes but its derivative diverges. The $2 \mathrm{D}$ waveguide is obtained by corrugating the upper line according to

$$
z=1+\varepsilon f(x)
$$

where $f(x)$ is a periodic function of period $2 \pi$ such that $f(x)>-1$ and $0 \leq \varepsilon \leq 1$. The curvilinear abscissa $s(x)$ of a point $Q$ on the corrugated line of coordinates $(x, z)$ where $z$ is given by 4

$$
s(x)=\int_{0}^{x} \sqrt{1+\varepsilon^{2} f^{\prime 2}\left(x^{\prime}\right)} d x^{\prime}
$$

Consider a ray which starts from $P_{0}=\left(x_{0}, 0\right)$ and reaches corrugated line at $Q=(x, z)$. After reflection the ray reaches the $x$ axis at the point $P_{1}=\left(x_{1}, 0\right)$. Keeping $P_{0}$ and $P_{1}$ fixed and letting $Q$ vary the path length $H$ of the segments $P_{0} Q$ and $Q P_{1}$ is a function of $s(x)$

$$
\begin{aligned}
H(s) & =h\left(x_{0}, s\right)+h\left(x_{1}, s\right) \\
h\left(x_{0}, s\right) & =\sqrt{\left(x-x_{0}\right)^{2}+(1+\varepsilon f(x))^{2}} \\
h\left(x_{1}, s\right) & =\sqrt{\left(x-x_{1}\right)^{2}+(1+\varepsilon f(x))^{2}}
\end{aligned}
$$

where $x=x(s)$ is the inverse of the function $s=s(x)$ defined by (4). Referring to Fig. 1 we define $\psi$ and $\psi^{\prime}$ the angles which the velocities $\mathbf{v}_{0}$ and $\mathbf{v}$ of the incoming and outgoing ray at $Q$ forms with the tangent $\tau$. The angles between the vectors $-\mathbf{v}_{0}$ and $\mathbf{v}$ and the normal $v$ at $Q$ are $\pi / 2-\psi$ and $\pi / 2-\psi^{\prime}$. As a consequence

$$
\begin{gathered}
\mathbf{v}_{0}=\frac{\left(x-x_{0}, 1+\varepsilon f(x)\right)^{T}}{h\left(x_{0}, s\right)} \quad \mathbf{v}=\frac{\left(x_{1}-x,-1-\varepsilon f(x)\right)^{T}}{h\left(x_{1}, s\right)} \\
\tau=\frac{\left(1, \varepsilon f^{\prime}(x)\right)^{T}}{\sqrt{1+\varepsilon^{2} f^{\prime 2}(x)}} \quad v=\frac{\left(\varepsilon f^{\prime}(x),-1\right)^{T}}{\sqrt{1+\varepsilon^{2} f^{\prime 2}(x)}}
\end{gathered}
$$

We denote with $\mathbf{v}_{1}$ the velocity of the ray reflected at $P_{1}$ and with $\theta_{0}$ and $\theta_{1}$ the angles $\mathbf{v}_{0}$ and $\mathbf{v}_{1}$ form with the positive
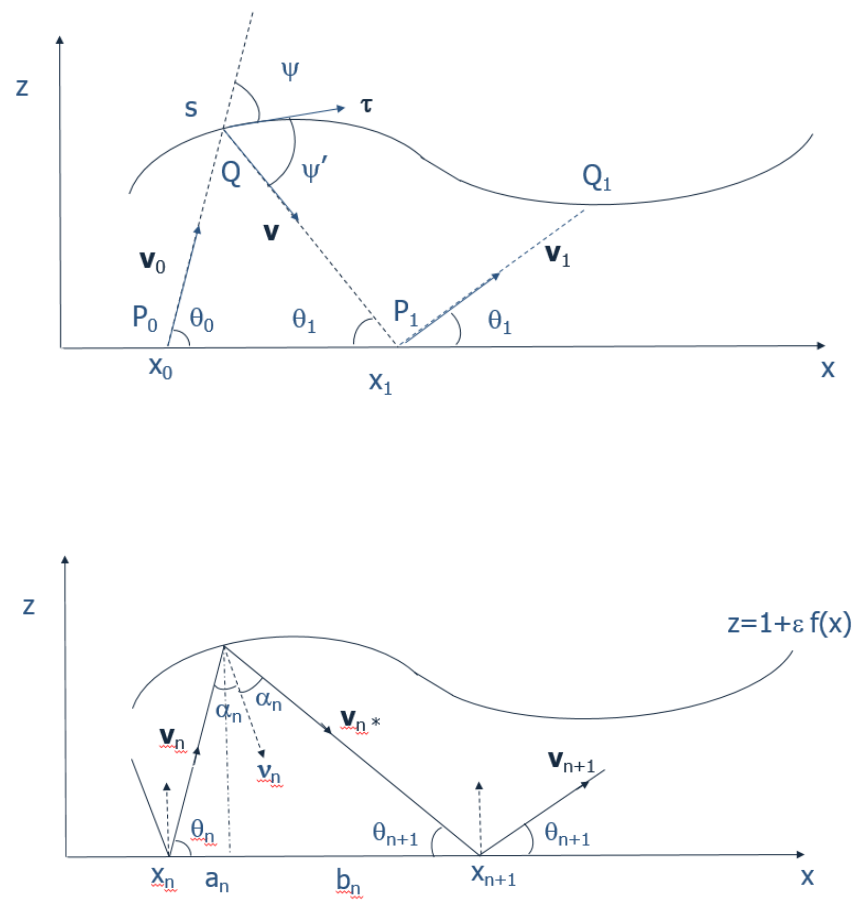

FIG. 1: Geometry of the reflections. On the right frame for $n=0$ we have $\alpha_{0}=\pi / 2-\psi$, where $\psi$ is defined on the left frame.

$x$ axis, see (1). The derivatives of $h\left(x_{1}, s\right)$ and of $h\left(x_{1}, s\right)$ are given by

$$
\begin{aligned}
& \frac{\partial}{\partial s} h\left(x_{0}, s\right)=\frac{x-x_{0}+\left(1+\varepsilon(f(s)) \varepsilon f^{\prime}(x)\right.}{h\left(x_{0}, s\right) \sqrt{1+\varepsilon^{2} f^{\prime 2}(x)}=}=\mathbf{v}_{0} \cdot \tau=\cos \psi \\
& \frac{\partial}{\partial x_{0}} h\left(x_{0}, s\right)=\frac{x_{0}-x}{h\left(x_{0}, s\right)}=-v_{x 0}=-\cos \theta_{0} \\
& \frac{\partial}{\partial s} h\left(x_{1}, s\right)=\frac{x-x_{1}+\left(1+\varepsilon(f(s)) \varepsilon f^{\prime}(x)\right.}{h\left(x_{1}, s\right) \sqrt{1+\varepsilon^{2} f^{\prime 2}(x)}}= \\
& \frac{\partial}{\partial x_{1}} h\left(x_{1}, s\right)=\frac{x_{1}-x}{h\left(x_{0}, s\right)}=v_{x 1}=\cos \theta_{1} \\
&
\end{aligned}
$$

The stationary point $H$ with respect to $s$, when $x_{0}$ and $x_{1}$ are kept fixed, is met for $s=s_{*}\left(x_{1}, x_{2}\right)$ where $\psi=\psi^{\prime}$, which corresponds to the the reflection condition. We introduce the function $F\left(x_{0}, x_{1}\right)$ equal to $H$ at the stationary point $s=$ 
$s_{*}\left(x_{0}, x_{1}\right)$ and compute its differential

$$
\begin{gathered}
F\left(x_{0}, x_{1}\right)=h\left(x_{0}, x_{1}, s_{*}\right)+h\left(x_{0}, x_{1}, s_{*}\right) \\
d F\left(x_{0}, x_{1}\right)=-v_{x 0} d x_{0}+v_{x 1} d x_{1}
\end{gathered}
$$

Equation (8) shows that $F\left(x_{0}, x_{1}\right)$ is the generating function of the canonical transformation $M$ from $\left(x_{0}, v_{x 0}\right)$ to $\left(x_{1}, v_{x 1}\right)$. After $n$ iterations the phase space point $\left(x_{n}, v_{x n}\right)$ is reached and is mapped into $\left(x_{n+1}, v_{x n+1}\right)$ by $M$. In the physical space the ray issued from the point $P_{n}=\left(x_{n}, 0\right)$ with horizontal velocity $v_{x n}$ is reflected at $Q_{n}=\left(x_{n}, 1+\varepsilon f\left(x_{n}\right)\right)$ and reaches $P_{n+1}=$ $\left(x_{n+1}, 0\right)$ where the horizontal velocity after reflection is $v_{x n+1}$ see figure 1 .

In order to obtain the map we first compute the time $\tau$ needed for the transit between $P_{n}$ and $Q_{n}$. Then we determine the horizontal component of the velocity $v_{x n+1}$ of the ray outgoing from $P_{n+1}$ which is equal to $v_{x *}$, where the velocity $\mathbf{v}_{*}$ of the ray from $Q_{n}$ to $P_{n+1}$ is determined by the reflection condition $\mathbf{v}_{*}=\mathbf{v}_{n}-2 v\left(v \cdot \mathbf{v}_{\mathbf{n}}\right)$ and the normal of the guide at $Q_{n}$ is given by (6). Finally $x_{n+1}-x_{n}$ is given by $v_{x n} \tau+v_{x n+1} \tau^{\prime}$ where the transit time from $\tau^{\prime}$ from $Q_{n}$ to $P_{n+1}$ is given by $v_{z n+1} \tau^{\prime}=v_{z n} \tau$.

$$
\begin{aligned}
& v_{z n} \tau=1+\varepsilon f\left(x_{n}+\tau v_{x n}\right), \quad v_{z n}=\sqrt{1-v_{x n}^{2}} \\
& v_{x n+1}=v_{x n}-2 v_{x}\left(v \cdot \mathbf{v}_{\mathbf{n}}\right)= \\
& =v_{x n}-2 \frac{\varepsilon^{2} f^{\prime 2} v_{x n}-\varepsilon f^{\prime} v_{z n}}{1+\varepsilon^{2} f^{\prime 2}} \\
& f^{\prime}=f^{\prime}\left(x_{n}+\tau v_{x n}\right) \\
& x_{n+1}=x_{n}+\tau\left(v_{x n}+v_{x n+1} \frac{v_{z n}}{v_{z n+1}}\right) \\
& v_{z n+1}=\sqrt{1-v_{x n+1}^{2}}
\end{aligned}
$$

The map $\left(x_{n+1}, v_{x n+1}\right)=M\left(x_{n}, v_{x n}\right)$ is symplectic since it is implicitly defined by the generating function $F\left(x_{n}, x_{n+1}\right)$ according to $v_{x n}=-\partial F / \partial x_{n}$ and $v_{x n+1}=\partial F / \partial x_{n+1}$. The computation of the tangent map, is given in the Appendix.

\section{THE 3D WAVE-GUIDE}

In this case we consider two planes $z=0$ and $z=1$ and the ray velocity is the unit vector $\mathbf{v}$ which can be written in Cartesian coordinates according to

$$
\mathbf{v}=\left(v_{x}, v_{y}, v_{z}\right) \quad v_{z}=\sqrt{1-v_{x}^{2}+v_{y}^{2}}
$$

With $\mathbf{v}$ we denote the velocity of the ray reflected by the lower plane $z=0$ so that $v_{z}>0$. The time $\tau$ between a reflection on the lower and upper plane is $\tau v_{z}=1$ so that, choosing $\left(x, y, v_{x}, v_{y}\right)$ as phase space coordinates, the map between two consecutive reflections reads

$$
\begin{aligned}
& v_{x n+1}=v_{x n} \\
& v_{y n+1}=v_{y n} \\
& x_{n+1}=x_{n}+2 \tau v_{x, n}=x_{n}+2 \frac{v_{x n}}{v_{z} n} \\
& y_{n+1}=y_{n}+2 \tau v_{y, n}=x_{n}+2 \frac{v_{y n}}{v_{z} n}
\end{aligned}
$$

If the map is defined on $\mathbb{T}^{2}([-\pi, \pi]) \times[-1,1]^{2}$, then $v_{v}, v_{y}$ are the action variables and the interpolating Hamiltonian is $H=-2 \sqrt{1-v_{x}^{2}-v_{y}^{2}}$. In the 3D waveguide the plane $z=1$ is corrugated, namely it is replaced by the surface

$$
z=z(x, y)=1+\varepsilon f(x, y)
$$

where $f(x, y)$ is a periodic function in $x$ and $y$ with period $2 \pi$ and $f>-1$ with $0<\varepsilon<1$. As a consequence the map describing the rays propagation can be defined on $\mathbb{T}^{2}([-\pi, \pi]) \times[-1,1]^{2}$. As in the $2 \mathrm{D}$ case we consider the sequence of points $P_{0}, P_{1}, \ldots, P_{n}, \ldots$ on the $z=0$ plane. We denote by $Q_{n}$ the point on the upper corrugated plane hit by the ray issued from $P_{n}$, which after reflection reaches the $z=0$ plane at $P_{n+1}$. The sequence points $P_{0}, P_{1}^{\prime}, \ldots, P_{n}^{\prime}, \ldots$ on the torus $\mathbb{T}^{2}$ is simply obtained taking the modulus with respect to $2 \pi$ so that $\left(x_{n}^{\prime}, y_{n}^{\prime}\right) \in[-\pi, \pi]$. Letting $v(\mathbf{x}, \mathbf{y})$ be the normal at the corrugated surface at the reflection point $Q=(x, y, z)=1+\varepsilon f(x, y)$, which explicitly reads

$$
v(x, y)=\frac{\left(\varepsilon f_{x}, \varepsilon f_{y},-1\right)^{T}}{\sqrt{1+\varepsilon^{2}\left(f_{x}^{2}+f_{y}^{2}\right)}}
$$

The map $\left(x_{n+1}, y_{n+1}, v_{x n+1}, v_{y n+1}\right)=M\left(x_{n}, y_{n}, v_{x n}, v_{y n}\right)$ specifying the ray trajectory is given by

$$
\begin{aligned}
& \tau v_{z n}=1+\varepsilon f\left(x_{n}+\tau v_{x n}, y_{n}+\tau v_{y n}\right) \\
& v_{z n}=\sqrt{1-v_{x n}^{2}-v_{y n}^{2}} \\
& v_{x n+1}=v_{x n}-2 v_{x}\left(v \cdot \mathbf{v}_{n}\right) \quad v=v\left(x_{n}+\tau v_{x n}, y_{n}+\tau v_{y n}\right) \\
& v_{y n+1}=v_{y n}-2 v_{y}\left(v \cdot \mathbf{v}_{\mathbf{n}}\right) \\
& x_{n+1}=x_{n}+\tau\left(v_{x n}+v_{x n+1} \frac{v_{z n}}{v_{z n+1}}\right) \\
& y_{n+1}=y_{n}+\tau\left(v_{y n}+v_{y n+1} \frac{v_{z n}}{v_{z n+1}}\right) \\
& v_{z n+1}=\sqrt{1-v_{x n+1}^{2}-v_{y n+1}^{2}}
\end{aligned}
$$

The first equation implicitly defines the propagation time $\tau$ from $P_{n}$ to $Q_{n}$, the remaining equations define the map $M$ 
which is symplectic. Indeed starting from the Fermat variational principle it is shown in appendix B that this map is obtained from a generating function $F\left(x_{n}, y_{n}, x_{n+1}, y_{n+1}\right)$. We do not quote in this case the expression of the tangent map since it is rather involved.

\section{DYNAMICAL INDICATORS}

We will present a numerical analysis of the 2D and 4D symplectic maps whose orbits describe the propagation of a ray in a $2 \mathrm{D}$ and $3 \mathrm{D}$ periodic waveguide. The stability analysis is intended to discriminate the regions of regular and chaotic motions using the recently introduced fast stability indicators, denominated Lyapunov and Reversibility error. The first one is closely related to fast Lyapunov indicator first proposed to analyze the growth of a small initial displacement. We shall not present a comparison with other fast indicators, because it has been performed for other models and since our indicators are independent from the initial displacement.

\section{A. Lyapounov error}

For a symplectic map in a phase space of dimension $2 d$ map the Lyapunov error (LE) describes the growth of an initial random displacement $\varepsilon \xi$ where $\xi \in \mathbb{R}^{\mathbf{2 d}}$ is a random vector with zero man and unit variance

$$
\langle\xi\rangle=0 \quad\left\langle\xi \xi^{T}\right\rangle=\mathrm{I}
$$

in the zero amplitude limit $\varepsilon \rightarrow 0$. Denoting with $\mathfrak{g}_{n}$ the random displacement after $n$ iteration and with $D M(x)$ the tangent map

$$
\begin{aligned}
& \Xi_{n}(\mathbf{x})=\lim _{\varepsilon \rightarrow 0} \frac{M^{n}(\mathbf{x}+\varepsilon \xi)-M^{n}(\mathbf{x})}{\varepsilon}=\mathrm{L}_{n}(\mathbf{x}) \xi \\
& \mathrm{L}_{n}(\mathbf{x})=D M^{n}(\mathbf{x})=\operatorname{DM}\left(\mathbf{x}_{n-1}\right) \mathrm{L}_{n-1}(\mathbf{x})
\end{aligned}
$$

The square of the Lyapunov error $E_{L n}(\mathbf{x})$ is defined as as the variance of of the random vector $\Xi_{n}$ or the trace of its covariance matrix

$$
E_{L n}^{2}(\mathbf{x})=\left\langle\Xi_{n} \cdot \Xi_{n}\right\rangle=\operatorname{Tr}\left(\Sigma_{n}^{2}\right) \quad \Sigma_{n}^{2}=\left\langle\Xi_{n} \Xi_{n}^{T}\right\rangle=\mathrm{L}_{n} \mathrm{~L}_{n}^{T}
$$

We might define the error $E_{L n}(\mathbf{x}, \eta)$ for a given initial displacement $\varepsilon \eta$ with $\|\eta\|=1$, when $\varepsilon \rightarrow 0$. Its logarithm is just the Fast Lyapunov Indicator. We observe that letting $\mathbf{e}_{j}$ be any orthonormal base the sum of $E_{L n}^{2}\left(\mathbf{x}, \mathbf{e}_{j}\right)$ extended to all the vectors of base is equal to $E_{L n}^{2}(\mathbf{x})$. Our definition is independent from the initial displacement. An expression for the Lyapunov error equivalent to (17) is given by

$$
E_{L n}^{2}(\mathbf{x})=\operatorname{Tr}\left(\mathrm{L}_{n}^{T}(\mathbf{x}) \mathrm{L}_{n}(\mathbf{x})\right)=\operatorname{Tr}\left(\left(D M^{n}(\mathbf{x})\right)^{T} D M^{n}(\mathbf{x})\right)
$$

The stability analysis is performed by fixing $n$ and observing the change of $E_{n}^{L}$ when $\mathbf{x}$ varies on a $2 D$ phase plane (or a
2D manifold). Oseledet theorem ${ }^{43}$ states that if $\mathbf{x}$ belongs to an ergodic component then $\left(\mathrm{L}_{n}^{T} \mathrm{~L}_{n}\right)^{1 / 2 n}$ has a limit $\mathrm{W} e^{\Lambda} \mathrm{W}^{T}$ independent from $\mathbf{x}$, where $\mathrm{W}$ is an orthogonal matrix, $\Lambda$ is diagonal and its entries $\lambda_{1} \geq \lambda_{2} \geq \ldots \geq \lambda_{2 d}$ are the Lyapunov exponents with $\lambda_{d+k}=-\lambda_{d-k+1}$ and $\lambda_{k} \geq 0$ for $1 \leq k \leq d$. As a consequence asymptotically $E_{L n}^{2} \simeq e^{2 n \lambda_{1}}+e^{2 n \lambda_{2}}+\ldots+$ $e^{2 n \lambda_{2 d}} \simeq e^{2 n \lambda_{1}}$ and more rigorously $\lim _{n \rightarrow \infty}\left(E_{L n}\right)^{1 / n}=\lambda_{1}$. However we are not interested in the $n \rightarrow \infty$ limit but rather on the dependence on $\mathbf{x}$ for a finite (possibly large) value of $n$, when $\mathbf{x}$ varies in phase space.

\section{B. Reversibility error}

We consider now the iteration of the map $n$ times followed by the iterations of the inverse map still $n$ times. Inserting a small additive noise at each forward and backward iteration, the final displacement with respect to the initial condition is a stochastic process, whose variance defines the square on the reversibility error (RE). More precisely letting $\mathbf{x}_{\varepsilon, 0}=\mathbf{x}$ be the initial conditions, we consider the noisy orbit

$$
\mathbf{x}_{\varepsilon, k}=M\left(\mathbf{x}_{\varepsilon, k-1}\right)+\varepsilon \xi_{k} \quad \Xi_{k}=\lim _{\varepsilon \rightarrow 0} \frac{\mathbf{x}_{\varepsilon, k}-\mathbf{x}_{k}}{\varepsilon}
$$

for $k=1, \ldots, n$. Starting from $\mathbf{x}_{\varepsilon, n}$ we consider the reversed noisy orbit

$$
\begin{gathered}
\mathbf{x}_{\varepsilon, n,-k}=M^{-1}\left(\mathbf{x}_{\varepsilon, n-k+1}\right)+\varepsilon \xi_{-\mathbf{k}} \\
\Xi_{n,-k}=\lim _{\varepsilon \rightarrow 0} \frac{\mathbf{x}_{\varepsilon, n,-k}-\mathbf{x}_{n-k}}{\varepsilon}
\end{gathered}
$$

The random vector $\Xi_{k}$ satisfies a linear recurrence with initial condition $\Xi_{0}=\mathbf{0}$ whereas $\Xi_{n,-k}$ satisfies another recurrence initialised by $\Xi_{n, 0}=\Xi_{n}$, sec ${ }^{36137}$ for an explicit expression. We choose the random vectors $\xi_{\mathbf{k}}$ and $\xi_{-\mathbf{k}^{\prime}}$ independent for $k, k^{\prime}>0$

$$
\begin{aligned}
\left\langle\xi_{k} \xi_{k^{\prime}}^{T}\right\rangle & =\left|\delta_{k, k^{\prime}} \quad\left\langle\xi_{-k} \xi_{-k^{\prime}}^{T}\right\rangle=\right| \delta_{k, k^{\prime}} \\
\left\langle\xi_{-k} \xi_{k^{\prime}}^{T}\right\rangle & =\left\langle\xi_{k} \xi_{-k^{\prime}}^{T}\right\rangle=0
\end{aligned}
$$

Letting $\Xi_{R n}=\Xi_{n,-n}$ be the stochastic displacement with respect to the initial condition $\mathbf{x}$ after reversing the orbit and $\Sigma_{R n}^{2}(\mathbf{x})$ the corresponding covariance matrix we define the reversibility error $E_{R}(\mathbf{x})$ according to

$$
\begin{aligned}
& E_{R n}^{2}(\mathbf{x})=\left\langle\Xi_{R n} \cdot \Xi_{R n}\right\rangle=\operatorname{Tr}\left(\Sigma_{R n}^{2}(\mathbf{x})\right) \\
& \Sigma_{R n}^{2}(\mathbf{x})=\left\langle\Xi_{R n}\left(\Xi_{R n}\right)^{T}\right\rangle
\end{aligned}
$$

The covariance matrix that $\Sigma_{R n}^{2}(\mathbf{x})$ can be expressed in terms of the tangent maps $D M^{k}(\mathbf{x})$ and their inverses $D M^{-k}(\mathbf{x})$. We do not quote their expression, which can be found in $\frac{35136}{3}$ since it can be proved ${ }^{38}$ that for a symplectic map $M$ in $\mathbb{R}^{2 d}$ the error 
$\mathrm{RE}$ is related to LE by the following expression

$$
\begin{aligned}
E_{R n}^{2}(\mathbf{x}) & =E_{L 0}^{2}(\mathbf{x})+E_{L n}^{2}(\mathbf{x})+2 \sum_{k=1}^{n-1} E_{L k}^{2}(\mathbf{x}) \\
E_{L 0}^{2} & =\operatorname{Tr}(\mathrm{I})=2 d
\end{aligned}
$$

or by the recurrence $E_{R n}^{2}=E_{R n-1}^{2}+E_{L n}^{2}+E_{L n-1}^{2}$ initialized by $E_{R 0}^{2}=0$ and $E_{L 0}^{2}=2 d$.

We finally define the reversibility error due to to round off (REM). Letting $M_{\varepsilon}(\mathbf{x})$ the map computed with a finite precision $\varepsilon$ (typically in the 8 bytes representation of reals $\varepsilon \simeq 10^{-17}$ ) and with $M_{\varepsilon}^{-1}$ the inverse of $M$ computed with finite accuracy, so that $M_{\varepsilon}^{-1}\left(M_{\varepsilon}(\mathbf{x})\right) \neq \mathbf{x}$, the modified reversibility error (REM) is defined according to

$$
E_{\mathrm{REM} n}(\mathbf{x})=\frac{\left\|M_{\varepsilon}^{-n}\left(M_{\varepsilon}^{n}(\mathbf{x})\right)-\mathbf{x}\right\|}{\varepsilon}
$$

Letting $\mathbf{x}_{\varepsilon, n}=M_{\mathcal{\varepsilon}}\left(\mathbf{x}_{\varepsilon, n-1}\right)$ be the orbit computed with round off, the local error $\left(M_{\varepsilon}\left(\mathbf{x}_{\varepsilon, n-1}\right)-M\left(\mathbf{x}_{\varepsilon, n-1}\right)\right) / \varepsilon$ is similar to a random vector, possibly correlated, if the map has a sufficiently high computational complexity, see $\sqrt{40 \mid 41}$. The difference with respect to the additive noise, we have considered to define RE, is that we have just a single realization. As a consequence even though the behaviour of RE and REM is similar, the last one exhibits large fluctuations, when $n$ varies. On the other side the computation of REM is really trivial since it requires a few lines of code, if the inverse map is available. This is the case of the reflection map for a waveguide. Indeed to reverse the evolution after $n$ iterations we must simply change the sign of the horizontal velocity components(s) and iterate again the same map $n$ times.

\section{Integrable and quasi-integrable maps}

The computation of LE and RE can be analytically performed for an integrable map. If the 2D map is a rotation $M(\mathbf{x})=\mathrm{R}(\omega) \mathbf{x}$ then $E_{L n}^{2}=2$ and $E_{R n}^{2}=4 n$. If the map $M$ is linearly conjugated to a rotation by $\mathbf{x}=\mathrm{TX}$ then the map in normal coordinates is $\mathrm{R}(\omega) \mathbf{X}$ and $M(\mathbf{x})=\mathrm{TR}(\omega) \mathrm{T}^{-1} \mathbf{x}$ in the given coordinates. The loss of symmetry in the coordinates $\mathbf{x}$ induces periodic oscillations $\left(E_{L n}^{2}=A+(2-A) \cos (\omega n)\right.$ with $A \geq 2$ and $E_{R n}$ has a linear growth with oscillations. A map $M(\mathbf{x})$ is integrable if there is a coordinate transformation $\mathbf{x}=T(\mathbf{X})$ such that the map becomes $N(\mathbf{X})=R(\Omega(J)) \mathbf{X}$ where the frequency depends on the action $J=\frac{1}{2}\|\mathbf{X}\|^{2}$. In this case $M(\mathbf{x})=T \circ N(\Omega(J)) \circ T^{-1}(\mathbf{x})$. The Lyapunov and reversibility errors LE and LE, depend on the initial condition and in the normal coordinates $\mathbf{X}$ they grow according to

$$
\begin{aligned}
& E_{L n}^{2}(\mathbf{X})=2+\left(2 J \Omega^{\prime}(J) n\right)^{2} \\
& E_{R n}^{2}(\mathbf{X}) 4 n+2\left(2 J \Omega^{\prime}(J)\right)^{2}\left(\frac{n^{3}}{3}+\frac{n}{6}\right)
\end{aligned}
$$

The Lyapunov error $L E$ in the coordinates $\mathbf{x}$ exhibits a linear growth with oscillations, due to the loss of rotational symme- try as in the linear case and we have

$$
\begin{array}{r}
E_{L n}^{2}(\mathbf{x})=\alpha_{0}(n)+n J \Omega^{\prime}(J) \alpha_{1}(n) \\
+\left(J \Omega^{\prime}(J) n\right)^{2} \alpha_{2}(n)
\end{array}
$$

where $J=\frac{1}{2}\left\|T^{-1}(\mathbf{x})\right\|^{2}$ and $\alpha_{k}$ are periodic functions of $n$ with frequency $\Omega(J)$. According to equation 23 the square of the reversibility error RE grows as $n^{3}$ with oscillations whose amplitude grows as $n^{2}$. If the map is quasi integrable as in the neighborhood of an elliptic fixed point at $\mathbf{x}_{*}$ with nonresonant frequency, then it is conjugated with its normal form up to a remainder which can be made exponentially small with $\sim e^{-a / r^{\alpha}}$ where $r=\left\|\mathbf{x}-\mathbf{x}_{*}\right\|$. Exponentially small estimates for the remainder can also be obtained for the error on the tangent map.

If the system has an hyperbolic point at $\mathbf{x}=(x, p)^{T}=\mathbf{0}$ and it is linear then $M(\mathbf{x})=\left(e^{-\lambda} x, e^{\lambda} p\right)^{T}$ and $E_{L n}^{2}=2 \operatorname{ch}(2 n \lambda) \sim$ $e^{2 n \lambda}$. If the system is non linear, in normal coordinates the map is $X_{n+1}=e^{-\lambda(X P)} X_{n}$ and $P_{n+1}=e^{\lambda(X Y)} P_{n}$ with initial condition $\mathbf{X}_{0}=(X, P)^{T}$. The Lyapunov error is

$$
E_{L n}^{2}(\mathbf{X})=e^{2 n \lambda}\left(1+n X P \lambda^{\prime}(X P)\right)^{2}\left(1+O\left(e^{-2 \lambda n}\right)\right)
$$

The square of LE near an elliptic equilibrium point for a generic symplectic map grows linearly with oscillations, the square of RE grows as $n^{3}$ with oscillations whose amplitude grows as $n^{2}$ so that asymptotically they become negligible. Near a hyperbolic point LE and RE have the same exponential growth. Finally REM behaves just as RE but exhibits large fluctuations. Right after the break up of the invariant curves surrounding an elliptic point sticky orbits are found and the indicators have initially a power law growth followed by an exponential growth. We conclude that RE is the smoothest indicator, since LE can exhibit oscillations and REM is affected by large fluctuations. However if we choose a fixed value of $n$ large enough, the portraits all these indicators in a chosen plane of phase space are very similar.

\section{THE MODEL OF A 2D WAVEGUIDE}

We present first a numerical analysis of the orbits for the map of the $2 \mathrm{D}$ waveguide where the corrugation is given by

$$
f(x)=\cos (x)
$$

The phase space coordinates are $\left(x, v_{x}\right)$ and the map is defined on the cylinder $\mathbb{T}([-\pi, \pi]) \times[-1,1]$ where $\mathbb{T}(([-\pi, \pi])$ is the interval $[-\pi, \pi]$ with identified ends. The map has an elliptic fixed point at $x=0, v_{x}=0$ where $f(x)$ has a maximum and is approximated by $f(x)=1-\frac{1}{2} x^{2}+O\left(x^{4}\right)$. We expand the map retaining only the linear terms in $x, v_{x}$. The first equation in (9) gives $\tau=1+\varepsilon$ neglecting second order terms in $x$ and $v_{x}$. Since $f^{\prime}(x)=-x+O\left(x^{3}\right)$ in the second equation the square of $f^{\prime}$ are neglected and $v_{z n}$ is replaced with 1 . Accordingly the second and third equations become

$$
\begin{aligned}
v_{x n+1} & =v_{x n}-2 \varepsilon\left(x_{n}+\tau v_{x n}\right) \\
x_{n+1} & =x_{n}+\tau\left(v_{x n}+v_{x n+1}\right)
\end{aligned}
$$


Letting $M(\mathbf{x})=\mathrm{Lx}$ be the linear map we have

$$
\mathrm{L}=\left(\begin{array}{cc}
1-2 \varepsilon \tau & 2 \tau-2 \varepsilon \tau^{2} \\
-2 \varepsilon & 1-2 \varepsilon \tau
\end{array}\right)
$$

so that $\operatorname{det} L=1$ and $\frac{1}{2} \operatorname{Tr} L=1-2 \varepsilon \tau$. The map is conjugated to a rotation $\mathrm{L}=\mathrm{VR}(\omega) \mathrm{V}^{-1}$ where $\sin ^{2}(\omega / 2)=\tau \varepsilon=$ $\varepsilon(1+\varepsilon)$. In this case we have $\mathrm{L}_{n}=\mathrm{L}^{n}$. Close to $x=0$ the profile $z=1+\varepsilon-\varepsilon x^{2} / 2$ is a concave mirror and the elliptical trajectories in phase space $\left(x, v_{x}\right)$ correspond to caustics in configuration space, namely the plane $(x, z)$ where the rays propagate. Close to $x=\pi$ the profile is approximated by $z=1-\varepsilon+\frac{1}{2} \varepsilon(x-\pi)^{2}+O(x-\pi)^{4}$ so that $\tau=1-\varepsilon$ and the linearized map is $\left(x_{n+1}, v_{x n+1}\right)^{T}=\mathrm{L}\left(x_{n}, v_{x n}\right)^{T}$, where $\mathrm{L}$ is given by (29) with $\varepsilon \rightarrow-\varepsilon$. The map $L$ is conjugated to a hyperbolic rotation $\mathrm{R}_{H}(\alpha)$ where $\operatorname{sh}^{2}(\alpha / 2)=\varepsilon \tau=\varepsilon(1-\varepsilon)$. Near $x=\pi$ the wave guide corresponds to an optical system given by a plane and convex mirror 1 .

The map defined by (9) was computed by solving the equation for $\tau$ with the bisection method initialized by $\tau_{1}=$ $0.5 / \sqrt{1-v_{x n}^{2}}$ and $\tau_{2}=1.5 / \sqrt{1-v_{x n}^{2}}$. The convergence is achieved also when $v_{x n}$ approaches 1 or -1 . The number of iterations to reach machine accuracy varies between 40 and 60 . Newton's method is faster and machine accuracy is reached in less 10 iterations, but convergence problems are met when $v_{x n}$ approaches 1 or -1 . We have also computed the tangent map and the explicit expression is written in Appendix A. Writing the implicit equation $G\left(x_{n}, v_{x n}, \tau\right)=0$, the solution is not defined if $\partial G / \partial \tau=0$ a condition numerically never met. In figures 2, 3 we compare the phase portraits with the colour plots of LE, RE and REM for two different values of the corrugation amplitude $\varepsilon=0.1,0.2$ respectively and orbits length $N=200$. The correspondence is quite good and RE appears to be the smoothest indicator since it is free from oscillations and fluctuations when $n$ varies. Increasing $N$ does not change the plots significantly. Higher values of $N$ are needed if one wishes to observe details in small regions where the transition from ordered to chaotic orbits occurs.

\section{THE 3D MODEL}

For the 3D waveguide we choose the corrugated profile according to

$$
f(x, y)=\cos x \cos y=\frac{1}{2} \cos (x+y)+\frac{1}{2} \cos (x-y)
$$

We remark first that for initial conditions $y_{0}=v_{y 0}=0$ we are back to the $2 \mathrm{D}$ case. If we choose $x_{0}=y_{0}$ and $v_{x 0}=v_{y 0}$ the ray propagates in the $x=y$ plane as for $2 \mathrm{D}$ waveguide. After a rotation of $\pi / 4$ the ray propagates on the $x^{\prime}, z$ plane where $x^{\prime}=\sqrt{2} x, v_{x}^{\prime}=\sqrt{2} v_{x}$, the corrugation function depends only on $x^{\prime}$ as $f=\frac{1}{2}\left[\cos \left(\sqrt{2} x^{\prime}\right)+1\right]$ and its period is $\sqrt{2} \pi$.

When the ray does not propagate in a plane the simplest case to analyze corresponds to the almost vertical propagation of the ray close to to a critical point $\left(x_{c}, y_{c}\right)$ of the corrugation function, where $\operatorname{grad} f=0$. The fixed point of the map $\left(x_{c}, y_{c}, v_{x}=0, v_{y}=0\right)$ is elliptic if $f$ has a maximum at $\left(x_{c}, y_{c}\right)$ and the corrugated surface near $\left(x_{c}, y_{c}\right)$ behaves as a concave mirror.

If $f$ has a minimum at $\left(x_{c}, y_{)}\right.$then the surface behaves as a convex mirror and and the critical point of the map is hyperbolic. For our model near $x=y=0$ we have $f \simeq 1-\frac{1}{2}\left(x^{2}+\right.$ $\left.y^{2}\right)$. The linearized map becomes

$$
\begin{aligned}
v_{x n+1} & =v_{x n}-2 \varepsilon\left(x_{n}+\tau v_{x n}\right) \\
x_{n+1} & =x_{n}+\tau\left(v_{x n}+v_{x n+1}\right) \\
v_{y n+1} & =v_{y n}-2 \varepsilon\left(y_{n}+\tau v_{y n}\right) \\
y_{n+1} & =y_{n}+\tau\left(v_{y n}+v_{y n+1}\right)
\end{aligned}
$$

where $\tau=1+\varepsilon$. Since $\tau$ is constant in this approximation the maps in the $\left(x, v_{x}\right)$ and $\left(y, v_{y}\right)$ phase planes decouple and each of them is area preserving. The linear frequencies are equal and given by $\sin ^{2}(\omega / 2)=\varepsilon \tau$ as for the $2 \mathrm{D}$ case. The degeneracy can be removed choosing for instance $f(x, y)=\cos x+A \cos y$.

Near $x=0, y=\pi$ we have $f \simeq 1+\frac{1}{2}\left(x^{2}+(y-\pi)^{2}\right)$ and the fixed point of the map is hyperbolic. More generally $(0,0),( \pm \pi, \pm \pi),( \pm \pi, \mp \pi)$ are elliptic and $(0, \pm \pi),( \pm \pi, 0)$ are hyperbolic. We do not examine the projections of individual orbits on 2D phase space planes nor on 3D hyperplanes. Close to the elliptic fixed points single 2D tori are recognisable in the projections on 3D hyperplanes and even 2D phase space planes, but the projection of several orbits does not provide any useful information. For this reason we show here the plots of our short term indicators LE, RE and REM of orbits whose initial points belong to $2 \mathrm{D}$ phase planes.

The plots are obtained by computing our indicators for initial conditions $\left(x_{0}, v_{x 0}, y_{0}, v_{y 0}\right)$ in a family of $2 \mathrm{D}$ phase planes where $y_{0}$ and $v_{y 0} / v_{x 0}$ are kept fixed. Letting $v_{x 0}=v_{0} \cos \left(\phi_{0}\right)$ and $v_{y 0}=v_{0} \sin \left(\phi_{0}\right)$ we compare the errors for fixed values of $y_{0}, \phi_{0}$ letting $x_{0}, v_{0}$ which vary in $[-\pi, \pi] \times[-1,1]$, where we choose a regular grid of $N_{g} \times N_{g}$ points. We have fixed the corrugation amplitude at $\varepsilon=0.1$ because there is a good balance between regular and chaotic regions. In figures 4 . 5. 6 we compare the errors LE, RE, REM in a logarithmic color scale by choosing and $y_{0}=0 \phi_{0}=\pi / 4, \pi / 2,3 \pi / 4$. We have computed LE by using the shadow orbit to evaluate the tangent map, namely we have replaced the partial derivatives $\partial M_{i} / \partial x_{j}$ by finite differences, avoiding the cumbersome analytic evaluation. To this and we have chosen four different initial conditions $\mathbf{x}_{0}+\mathbf{e}_{j} \delta$ for $\leq j \leq 4$ where $\mathbf{e}_{j}$ are the orthonormal base vectors in $\mathbb{R}^{4}$ namely $\left(\mathbf{e}_{j}\right)_{k}=\delta_{j_{k}}$. The tangent map is approximated by

$$
D M^{n}\left(\mathbf{x}_{0}\right) \mathbf{e}_{j} \simeq \mathbf{w}_{j}(n) \equiv \frac{M^{n}\left(\mathbf{x}_{0}+\mathbf{e}_{j} \delta\right)-M^{n}\left(\mathbf{x}_{0}\right)}{\delta}
$$

so that the Lyapunov error becomes

$$
E_{L n}^{2} \simeq \sum_{j=1}^{4}\left\|\mathbf{w}_{j}(n)\right\|^{2}
$$


the discrepancy is of order $\delta$ and the choice $\delta=10^{-14}$ was made, using double precision accuracy.

For $\phi_{0}=0$ we recover the plot computed with the tangent map for the $2 \mathrm{D}$ waveguide since the ray propagates in the $x, z$ plane, but only if the sum in equation (34) runs only up to 2 (corresponding to the phase space coordinates to $x, v_{x}$ ). This is equivalent to define LE by replacing $D M^{n}$ with its first $2 \times 2$ block, which corresponds to tangent map for the 2D map. Indeed even though the orbits of the 2D and 4D map are the same for initial conditions $y_{0}=v_{y 0}=0$, the components of $4 \mathrm{D}$ tangent map, not belonging the $2 \times 2$ block, are non zero. On the contrary REM does not change with respect to $2 \mathrm{D}$ map, because the $\left(x, v_{x}\right)$ plane is invariant. To recover agreement between REM and RE, when LE is computed according to 34, which corresponds to the trace of $\left(D M^{n}\right)^{T} D M^{n}$, one can add a small random displacement of amplitude $\delta$ before reversing the orbit, in order to bring the orbit out of the invariant plane. When there is no invariant plane the agreement between REM and RE is recovered without any random kick. In figure (7) (a)-(c) we show the plot for $\phi \simeq \pi / 2$ and $y_{0}=0$. The regions of regular motion correspond to neighbourhood of elliptic points of the resonant structures, the regions of chaotic motion to to the separatrices joining the hyperbolic points. In addition the double resonances, due to single resonances intersection in action space, create other chaotic regions. The Fourier analysis might be used to classify the resonances because the resonant perturbation theory cannot be easily developed.

\section{SHANNON CHANNEL CAPACITY}

The possibility of computing fast stability indicators allows to establish interesting properties that depend on them. Among others, in information theory it is important to introduce the so called Shannon-Hartley channel capacity

$$
C=\lim _{t \rightarrow \infty} \frac{1}{t} \log _{2}\left(\frac{Y}{X}\right),
$$

where the time-dependent relation between input $(X)$ and output $(Y)$ describes the time evolution of the transfer process taking the message from transmitter to receiver. Equation (40) gives the maximum (ideal) information bit datarate achievable in a physical system that carry electric signals/electromagnetic waves. In the high-frequency asymptotics, the propagation of waves through the corrugated channel in Fig. 11 has an isomorphism with classical ray trajectories underlying waves that bounce around the channel, given by the symplectic map (9). Therefore, one can think that the maximum Lyapunov exponent has an isomorphism with the Shannon-Hartley channel capacity. This has been recognised in ${ }^{44}$ to be valid for any dynamical system, hence for a continuous time system. The relation between the channel capacity and the Lyapunov exponents of random matrices was first proposed by

$$
C=\lambda_{1}=\lim _{t \rightarrow \infty} \frac{1}{t} \log E_{L}(t)=\lim _{t \rightarrow \infty} \frac{1}{t} \log E_{R}(t)
$$

For recent works on the subject relating channel capacity to Lyapunov exponents and entropy see ${ }^{46}$ and ${ }^{47}$ Consequently, fast dynamical indicator 35136 allow an estimation of the channel capacity. For a map like the waveguide map depending on one parameter $\varepsilon$, given a finite number of iterations $n$ we introduce the sequences

$$
\begin{aligned}
C_{L n}(\mathbf{x}, \varepsilon) & =\frac{1}{n} \log E_{L n}(\mathbf{x}, \varepsilon) \\
C_{R n}(\mathbf{x}, \varepsilon) & =\frac{1}{n} \log E_{R n}(\mathbf{x}, \varepsilon)
\end{aligned}
$$

having the same limit $C(\mathbf{x}, \varepsilon)$ as $n \rightarrow \infty$. The channel capacities depend on the initial condition $\mathbf{x}$, however if $\mathbf{x}$ belongs to an ergodic component the result is the same for almost any choice of $\mathbf{x}$. For a parallel wave guide $\varepsilon=0$ the channel capacities $C_{L n}(\mathbf{x}, \varepsilon)$ and $C_{L n}(\mathbf{x}, \varepsilon)$ vanish asymptotically according to

$$
\begin{aligned}
& C_{L n}(\mathbf{x}, \varepsilon)=\frac{1}{n} \log n+O\left(n^{-1}\right) \\
& C_{R n}(\mathbf{x}, \varepsilon)=\frac{3}{2 n} \log n+O\left(n^{-1}\right)
\end{aligned}
$$

For a corrugated waveguide the phase plots of $C_{L n}(\mathbf{x}, \varepsilon)$ and $C_{R n}(\mathbf{x}, \varepsilon)$ for fixed $n$ of are the same as the plot of $E_{L n}$ and $E_{R n}$ in a logarithmic scale shown in the figures 2 and 3 for the $2 \mathrm{D}$ wave guide and 4 to 7 for the $4 \mathrm{D}$ waveguide, up to the constant factor $n^{-1}$.

The $2 \mathrm{D}$ ray reflection map for $\varepsilon \ll 1$ is almost integrable and exhibits two distinct regions delimited by a separatrix which can be approximated by

$$
v_{x}= \pm 2 \sqrt{\varepsilon} \cos \left(\frac{x}{2}\right)
$$

see figure 8 . For $\varepsilon \sim 0.1$ the separatrix becomes a thin stochastic layer, whose area grows by further increasing $\varepsilon$. We have compared the dependence on $n$ of the channel capacities $C_{L n}(\mathbf{x}, \varepsilon)$ and $C_{R n}(\mathbf{x}, \varepsilon)$ for one regular and two chaotic orbits, see figure 9 For the first orbit the channel capacity vanishes as $n^{-1} \log n$ whereas for the last two chaotic orbits a finite limit is approached.

In order to appreciate how the limit for $n \rightarrow \infty$ is reached and the dependence on the corrugated amplitude we compute the following phase space averages

$$
\begin{aligned}
C_{L n}(\varepsilon) & =\frac{1}{\mu_{L}(\mathscr{E})} \int_{\mathscr{E}} C_{L n}(\mathbf{x}, \varepsilon) d \mathbf{x} \\
C_{R n}(\varepsilon) & =\frac{1}{\mu_{L}(\mathscr{E})} \int_{\mathscr{E}} C_{R n}(\mathbf{x}, \varepsilon) d \mathbf{x}
\end{aligned}
$$

where $\mathscr{E}$ denotes the phase space and $\mu_{L}(\mathscr{E})$ its volume. We have analyzed the dependence on the corrugation amplitude $\varepsilon$ of the average channel capacities $C_{L n}(\varepsilon)$ and $C_{R n}(\varepsilon)$ for $n=100$ and $n=200$, see Figure 10 The channel capacity varies almost monotonically from 0 to 0.8 and corresponds to the phase space average of the maximum Lyapunov exponent, since for $n=200$ the asymptotic value appears to be reached. 
We have found the following quadratic fit with $\varepsilon$

$$
C(\varepsilon)=2.4 \varepsilon-1.6 \varepsilon^{2} \quad 0 \leq \varepsilon \leq \frac{1}{2}
$$

A similar analysis can be performed for the 4D map by computing the phase space average with a Monte Carlo sampling rather than on a regular grid as for the 2D map.

\section{CONCLUSIONS}

We have analyzed the propagation of a ray on $2 \mathrm{D}$ and $3 \mathrm{D}$ waveguide, given by two parallel lines or planes, one of which has a periodic corrugation. In both cases the ray reflection maps on the uncorrugated line or plane are symplectic. The stability properties are relevant for the long term propagation of the rays. Indeed in the chaotic regions a diffusion occurs preventing the coherent propagation of a signal. We have analyzed the reflection maps using the short time indicators recently proposed: the Lyapunov error LE, and the reversibility errors RE and REM. The square of LE is the trace of the covariance matrix of the displacement, after $n$ iterations of the map, induced by a small initial random displacement. The square of RE is the trace of the covariance matrix of the displacement from the initial condition, when the map is iterated forward and backward $n$ times, adding at each step a small random displacement. Replacing the random displacement with the round off allows to define a modified reversibility error REM, which is similar to RE, though affected by large fluctuations due to the absence of averaging. In the $2 \mathrm{D}$ case a good qualitative agreement with the phase portrait is found. For a fixed number $n$ of reflection of the rays on the uncorrugated plane, all the indicators exhibit a similar behaviour with a power law growth for regular orbits and exponential growth for chaotic orbits. For small corrugations the motion is regular almost everywhere except in the neighborhood of the separatrix, occurring when the rays hit a minimum of the corrugated line or surface, and for rays propagating almost parallel to the waveguide

For the orbits of the 4D map, describing the ray dynamics of the 3D waveguide, LE and RE provide a satisfactory stability portrait, just as REM. A stability analysis in this case cannot be performed by looking at the projection of orbits on a $2 \mathrm{D}$ phase plane. Altogether these indicators quantify separately the effect of small initial displacements, additive noise and round off. The limited computational load and the simplicity of implementation of these indicators make them a convenient tool also for a parametric study of $2 \mathrm{D}$ and $3 \mathrm{D}$ waveguides. We have also considered the relation of our indicators with the channel capacity $C$. Indeed the limit of $n^{-1} \log E_{L n}$ and $n^{-1} \log E_{R n}$ for $n \rightarrow \infty$ gives the maximum Lyapunov exponent which is isomorphic to the channel capacity. The dependence on $n$ for a given initial condition is consistent with theoretical estimates. The phase space average of the channel capacity is found to rise with the corrugation amplitude $\varepsilon$ following a quadratic law.

\section{DATA AVAILABILITY STATEMENT}

The data that support the findings of this study are openly available in https://github.com/gabrielegradoni/ WaveguideStability

\section{ACKNOWLEDGMENTS}

GG wishes to acknowledge the financial support of The Royal Society under the grant INF|R2|192066.

\section{APPENDIX A: TANGENT MAP FOR 2D WAVEGUIDE}

The equation which determines $\tau(x, v)$ is

$$
\begin{aligned}
G(x, v, \tau) & =1+\varepsilon f(x+\tau v)-\tau \sqrt{1-v^{2}}=0 \\
\frac{\partial \tau}{\partial x} & =-\frac{\frac{\partial G}{\partial x}}{\frac{\partial G}{\partial \tau}}=\frac{\varepsilon f^{\prime}}{\sqrt{1-v^{2}}-\varepsilon v f^{\prime}} \\
\frac{\partial \tau}{\partial v} & =-\frac{\frac{\partial G}{\partial v}}{\frac{\partial G}{\partial \tau}}=\frac{\varepsilon \tau f^{\prime}+\tau v / \sqrt{1-v^{2}}}{\sqrt{1-v^{2}}-\varepsilon v f^{\prime}}
\end{aligned}
$$

Letting $v_{x}=\cos \theta \equiv v$ and $v_{z}=\sin \theta \equiv \sqrt{1-v^{2}}$ the recurrence which determines the symplectic map $M=(x, v)$ is given by

$$
\begin{aligned}
& G\left(x_{n}, v_{n}, \tau\right)=0 \\
& v_{n+1}=v_{n}-2 \frac{\varepsilon^{2} f^{\prime 2} v_{n}-\varepsilon f^{\prime} \sqrt{1-v_{n}^{2}}}{1+\varepsilon^{2} f^{\prime 2}} \\
& x_{n+1}=x_{n}+\tau v_{n}+\tau \frac{\sqrt{1-v_{n}^{2}}}{\sqrt{1-v_{n+1}^{2}}} v_{n+1}
\end{aligned}
$$


The tangent map is given by

$$
\begin{aligned}
\frac{\partial v_{n+1}}{\partial x_{n}} & =\frac{2 \varepsilon f^{\prime \prime}}{\left(1+\varepsilon^{2} f^{\prime 2}\right)^{2}}\left[-2 \varepsilon v_{n} f^{\prime}+\sqrt{1-v_{n}^{2}}\left(1-\varepsilon^{2} f^{\prime 2}\right)\right] \times \\
& \times\left(1+v_{n} \frac{\partial \tau}{\partial x_{n}}\right) \\
\frac{\partial v_{n+1}}{\partial v_{n}} & =1-\frac{2 \varepsilon f^{\prime}}{1+\varepsilon^{2} f^{\prime 2}}\left(\varepsilon f^{\prime}+\frac{v_{n}}{\sqrt{1-v_{n}^{2}}}\right)+ \\
& +\frac{2 \varepsilon f^{\prime \prime}}{\left(1+\varepsilon^{2} f^{\prime 2}\right)^{2}}\left[-2 \varepsilon v_{n} f^{\prime}+\sqrt{1-v_{n}^{2}}\left(1-\varepsilon^{2} f^{\prime 2}\right)\right] \times \\
& \times\left(\tau+v_{n} \frac{\partial \tau}{\partial v_{n}}\right) \\
\frac{\partial x_{n+1}}{\partial x_{n}} & =1+\left(v_{n}+v_{n+1} \frac{\sqrt{1-v_{n}^{2}}}{\sqrt{1-v_{n+1}^{2}}}\right) \frac{\partial \tau}{\partial x_{n}}+ \\
& +\tau \frac{\sqrt{1-v_{n}^{2}}}{\left(1-v_{n+1}^{2}\right)^{3 / 2}} \frac{\partial v_{n+1}}{\partial x_{n}} \\
\frac{\partial x_{n+1}}{\partial v_{n}} & =\tau+\left(v_{n}+v_{n+1} \frac{\sqrt{1-v_{n}^{2}}}{\sqrt{1-v_{n+1}^{2}}}\right) \frac{\partial \tau}{\partial v_{n}}+ \\
& +\tau \frac{\sqrt{1-v_{n}^{2}}}{\left(1-v_{n+1}^{2}\right)^{3 / 2}} \frac{\partial v_{n+1}}{\partial v_{n}}- \\
& -\tau \frac{v_{n}}{\sqrt{1-v_{n}^{2}}} \frac{v_{n+1}}{\sqrt{1-v_{n+1}^{2}}}
\end{aligned}
$$

In all the previous formulae $f=f\left(x_{n}+\tau v_{n}\right)$ with $f^{\prime}=f^{\prime}\left(x_{n}+\right.$ $\left.\tau v_{n}\right)$ and $f^{\prime \prime}=f^{\prime \prime}\left(x_{n}+\tau v_{n}\right)$.

In order to check the limit $\varepsilon \rightarrow 0$ we recall that the map is given by

$$
\begin{aligned}
\tau \sqrt{1-v_{n}^{2}} & =1 \quad v_{n+1}=v_{n} \\
x_{n+1} & =x_{n}+2 \frac{v_{n}}{\sqrt{1-v_{n}^{2}}}
\end{aligned}
$$

In this case the tangent map is given by

$$
\begin{aligned}
\frac{\partial x_{n+1}}{\partial x_{n}} & =1 \\
\frac{\partial x_{n+1}}{\partial v_{n}} & =\frac{2}{\left(1-v_{n}^{2}\right)^{3 / 2}} \\
\frac{\partial v_{n+1}}{\partial x_{n}} & =0 \\
\frac{\partial v_{n+1}}{\partial v_{n}} & =1
\end{aligned}
$$

In our example we choose $f(x)$ periodic of period $2 \pi$ and scale the coordinates according to $x=2 \pi x^{\prime}$ so that the new tangent map is given by

$$
\begin{aligned}
\frac{\partial x_{n+1}^{\prime}}{\partial x_{n}^{\prime}} & =\frac{\partial x_{n+1}}{\partial x_{n}} \\
\frac{\partial x_{n+1}^{\prime}}{\partial v_{n}} & =\frac{1}{2 \pi} \frac{\partial x_{n+1}}{\partial v_{n}} \\
\frac{\partial v_{n+1}}{\partial x_{n}^{\prime}} & =2 \pi \frac{\partial v_{n+1}}{\partial x_{n}}
\end{aligned}
$$

\section{APPENDIX B. THE 3D WAVE-GUIDE}

The equation for the corrugates waveguide is given by equation (12) where $f(x, y)$ is a periodic function. The normal vector $v$ and the tangent vectors $\tau_{\mathbf{x}}, \tau_{\mathbf{y}}$ are given by

$$
\begin{aligned}
v(\mathbf{x}, \mathbf{y}) & =\frac{\left(\varepsilon f_{x}, \varepsilon f_{y},-1\right)^{T}}{\sqrt{1+\varepsilon^{2}\left(f_{x}^{2}+f_{y}^{2}\right)}} \\
\tau_{\mathbf{x}} & =\frac{\left(1,0, \varepsilon f_{x}\right)^{T}}{\sqrt{1+\varepsilon^{2} f_{x}^{2}}} \\
\tau_{\mathbf{x}} & =\frac{\left(0,1, \varepsilon f_{y}\right)^{T}}{\sqrt{1+\varepsilon^{2} f_{y}^{2}}}
\end{aligned}
$$

where $f_{x} \equiv \partial f / \partial x$ and $f_{y} \equiv \partial f / \partial y$. For an analogy with the 2D case we might introduce the curvilinear abscissas $s_{x}, s_{y}$ on the lines on the corrugated plane having $y$ fixed and $x$ fixed respectively

$$
\begin{aligned}
& s_{x}(x, y)=\int_{0}^{x} \sqrt{1+\varepsilon^{2} f_{x}^{2}\left(x^{\prime}, y\right)} d x^{\prime} \\
& s_{y}(x, y)=\int_{0}^{y} \sqrt{1+\varepsilon^{2} f_{y}^{2}\left(x, y^{\prime}\right)} d y^{\prime}
\end{aligned}
$$

so that, keeping y fixed, $d s_{x}=\sqrt{1+\varepsilon^{2} f_{x}^{2}(x, y)} d x$ and, keeping $\mathrm{x}$ fixed, $d s_{y}=\sqrt{1+\varepsilon^{2} f_{x}^{2}(x, y)} d y$. The transformation from $(x, y)$ to $\left(s_{x}, s_{y}\right)$ is invertible. Letting $P_{0}=\left(x_{0}, y_{0}, 0\right)$ be a point on the plane and $Q=(x, y, z(x, y))$ a point on the corrugated plane and $P_{1}=\left(x_{1}, y_{1}, 0\right)$ a new point on the plane we consider a ray whose path is $P_{0}, Q, P_{1}$. We denote with $\mathbf{v}_{0}$ and $\mathbf{v}$ the velocity of the ray directed from $P_{0}$ to $Q$ and from $Q$ to $P_{1}$. As for the $2 D$ case we keep $P_{0}$ and $P_{1}$ fixed letting $Q$ vary and consider the ray path length $H$ which depends on $Q$

$$
\begin{aligned}
H(x, y) & =h\left(x_{0}, y_{0}, x, y\right)+h\left(x_{1}, y_{1}, x, y\right) \\
h\left(x_{0}, y_{0}, x, y\right) & =\sqrt{\left(x-x_{0}\right)^{2}+\left(y-y_{0}\right)^{2}+(1+\varepsilon f(x, y))^{2}}
\end{aligned}
$$

The velocities $\mathbf{v}_{0}$ and $\mathbf{v}$, denoting for brevity $h_{0} \equiv h\left(x_{0}, y_{0}, x, y\right)$ and $h_{1} \equiv h\left(x_{1}, y_{1}, x, y\right)$, are given by

$$
\begin{aligned}
\mathbf{v}_{0} & =\frac{\left(x-x_{0}, y-y_{0}, 1+\varepsilon f(x, y)\right)^{T}}{h\left(x_{0}, y_{0}, x, y\right)} \\
\mathbf{v} & =\frac{\left(x_{1}-x_{0}, y_{1}-y_{0}, 1+\varepsilon f(x, y)\right)^{T}}{h\left(x_{1}, y_{1}, x, y\right)}
\end{aligned}
$$


We first compute the derivatives of $h_{0}$ and $h_{1}$ with respect to $x$ and $y$

$$
\begin{aligned}
& \frac{\partial}{\partial x} h_{0}=\frac{x-x_{0}+(1+\varepsilon f) \varepsilon f_{x}}{h_{0}}=\mathbf{v}_{0} \cdot \tau_{x} \sqrt{1+\varepsilon^{2} f_{x}^{2}} \\
& \frac{\partial}{\partial y} h_{0}=\frac{y-y_{0}+(1+\varepsilon f) \varepsilon f_{y}}{h_{0}}=\mathbf{v}_{0} \cdot \tau_{y} \sqrt{1+\varepsilon^{2} f_{y}^{2}} \\
& \frac{\partial}{\partial x} h_{1}=\frac{x-x_{1}+(1+\varepsilon f) \varepsilon f_{x}}{h_{1}}=-\mathbf{v} \cdot \tau_{x} \sqrt{1+\varepsilon^{2} f_{x}^{2}} \\
& \frac{\partial}{\partial y} h_{1}=\frac{y-y_{1}+(1+\varepsilon f) \varepsilon f_{y}}{h_{1}}=-\mathbf{v} \cdot \tau_{y} \sqrt{1+\varepsilon^{2} f_{y}^{2}}
\end{aligned}
$$

Choosing $h_{0}$ and $h_{1}$ a functions of $s_{x}, s_{y}$ rather than $x, y$ one has $\partial h_{0} / \partial s_{\alpha}=\mathbf{v}_{0} \cdot \tau_{\alpha}$ and and $\partial h_{1} / \partial s_{\alpha}=-\mathbf{v} \cdot \tau_{\alpha}$ for $\alpha=$ $x, y$. The function $H$ is stationary when $\mathbf{v}_{0} \cdot \tau_{\mathbf{x}}=\mathbf{v}_{\mathbf{0}} \cdot \tau_{\mathbf{x}}$ and $\mathbf{v}_{0} \cdot \tau_{\mathbf{y}}=\mathbf{v}_{\mathbf{0}} \cdot \tau_{\mathbf{y}}$ namely when the projection on the tangent plane of the incoming and outgoing ray velocities are equal. This corresponds to the reflection condition since the normal components of $\mathbf{v}_{0}$ and $\mathbf{v}$ are opposite. The derivatives with respect to $x_{0}, y_{0}$ and $x_{1}, y_{1}$ are given by

$$
\begin{array}{ll}
\frac{\partial h_{0}}{\partial x_{0}}=\frac{x_{0}-x}{h_{0}}=-v_{x 0} & \frac{\partial h_{0}}{\partial y_{0}}=\frac{y_{0}-y}{h_{0}}=-v_{y 0} \\
\frac{\partial h_{1}}{\partial x_{1}}=\frac{x_{1}-x}{h_{1}}=v_{x 1} & \frac{\partial h_{1}}{\partial y_{1}} h_{1}=\frac{y_{1}-y}{h_{1}}=-v_{y 1}
\end{array}
$$

Finally letting $\left(x_{*}, y_{*}\right)$ the point where $H$ is stationary and which depends on end points $\left(x_{0}, y_{0}\right)$ and $\left(x_{1}, y_{1}\right)$ we introduce the function $F$ defined as the value of $H$ evaluated at the stationary point and compute its differential

$$
\begin{aligned}
& F\left(x_{0}, y_{0}, x_{1}, y_{1}\right)=h\left(x_{0}, y_{0}, x_{*}, y_{*}\right)+h\left(x_{1}, y_{1}, x_{*}, y_{*}\right) \\
& d F=-v_{x 0} d x_{0}-v_{y 0} d y_{0}+v_{x} d x_{1}+v_{y 1} d y_{1}
\end{aligned}
$$

As a consequence $F$ is the generating function of a canonical transformation $M$ which maps $\left(x_{0}, v_{x 0}, y_{0}, v_{y 0}\right)$ into $\left(x_{1}, v_{x 1}, y_{1}, v_{y 1}\right)$. Denoting with $\mathbf{x}_{n}=\left(x_{n}, v_{x n}, y_{n}, v_{y n}\right)^{T}$ the phase space point reached after $n$ iterations of the symplectic map $M$, the recurrence from $\mathbf{x}_{n}$ to $\mathbf{x}_{n+1}$ is obtained first by computing the transit time $\tau$ from $P_{n}=\left(x_{n}, y_{n}\right)$ to the point $Q=\left(x_{*}, y_{*}\right)$ on the corrugated surface. Then we notice that the horizontal plane projections of the velocities $\mathbf{v}_{*}=\mathbf{v}_{n}-2 v\left(\mathbf{v}_{\mathbf{n}} \cdot v\right)$ and $\mathbf{v}_{n+1}$ of the ray reflected at $Q$ and $P_{n+1}=\left(x_{n+1}, y_{n+1}\right)$ are equal. Finally we determine the displacement from $P_{n}$ to $P_{n+1}$. The equations defining the recurrence from $\mathbf{x}_{n}$ to $\mathbf{x}_{n+1}$ are given by (14).

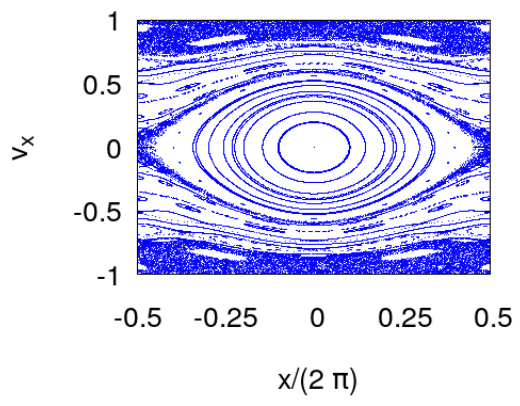

(a)

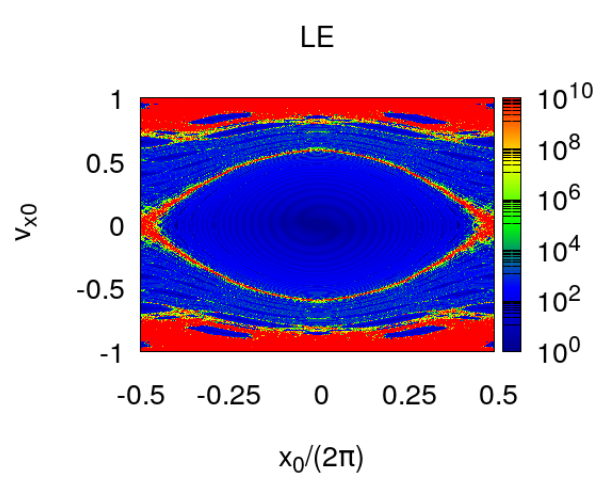

(b)

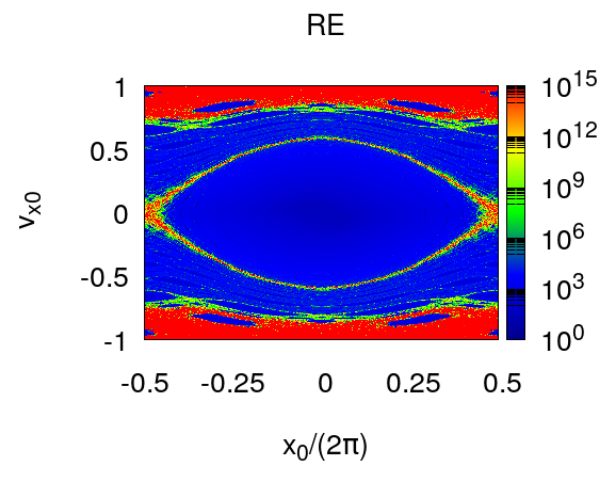

(c)

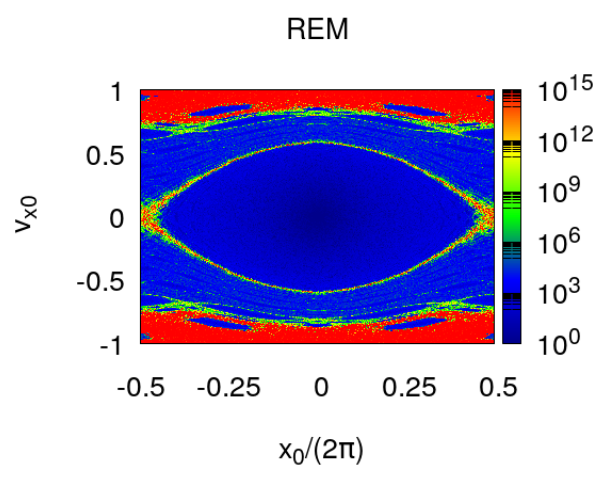

(d)

FIG. 2: From the top: (a) Phase portrait of the map for the 2D waveguide with corrugation $z=1+\varepsilon \cos (x)$ and $\varepsilon=0.1$. (b) Colour plot of LE for $N=200$ iterations of the map. (c) Plot of RE for $N=200$ iterations of the map. (d) Plot of REM for $N=200$ iterations of the map. 


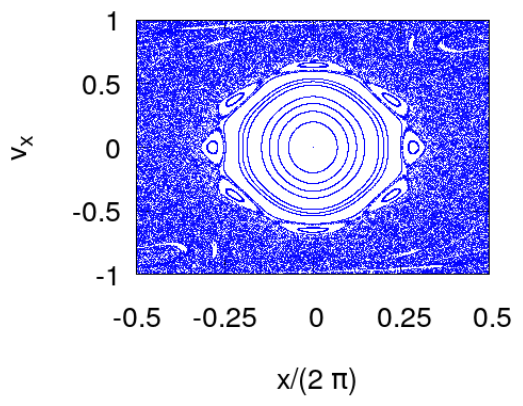

(a)

LE

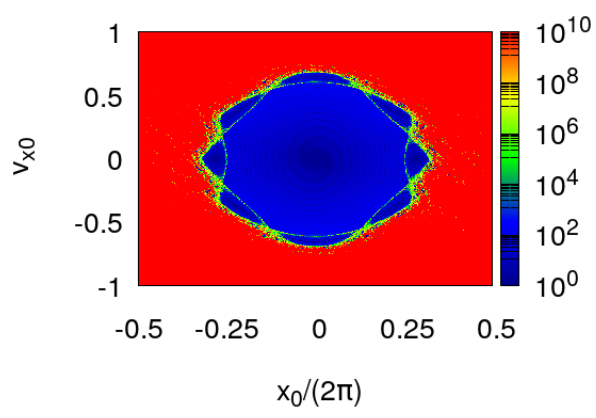

(b)

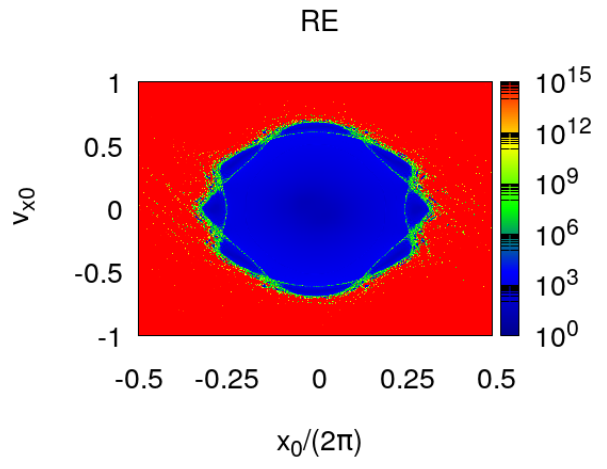

(c)

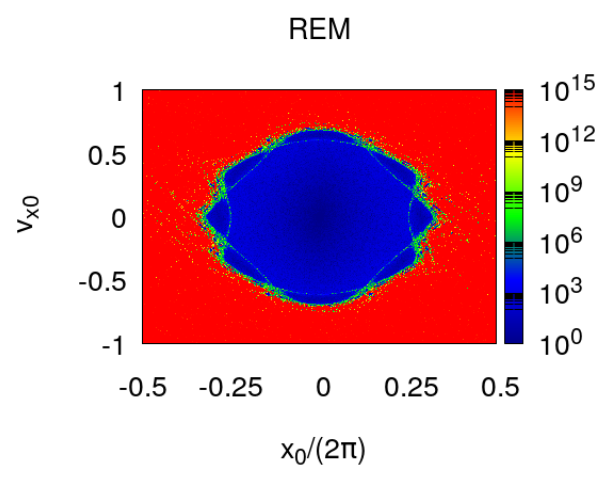

(d)

FIG. 3: From the top: (a) Phase portrait of the map for the 2D waveguide with corrugation $z=1+\varepsilon \cos (x)$ and $\varepsilon=0.2$.

(b) Colour plot of LE for $N=200$ iterations of the map. (c) Plot of RE for $N=200$ iterations of the map. (d) Plot of REM for $N=200$ iterations of the map.

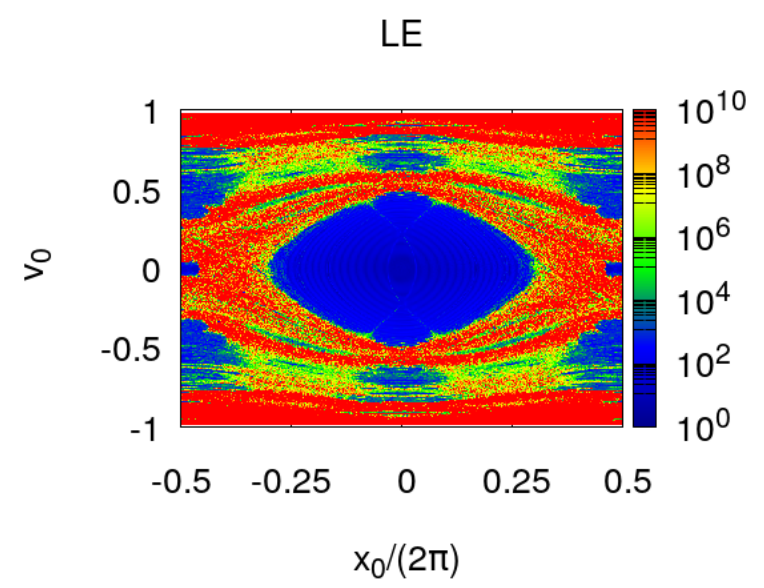

(a)

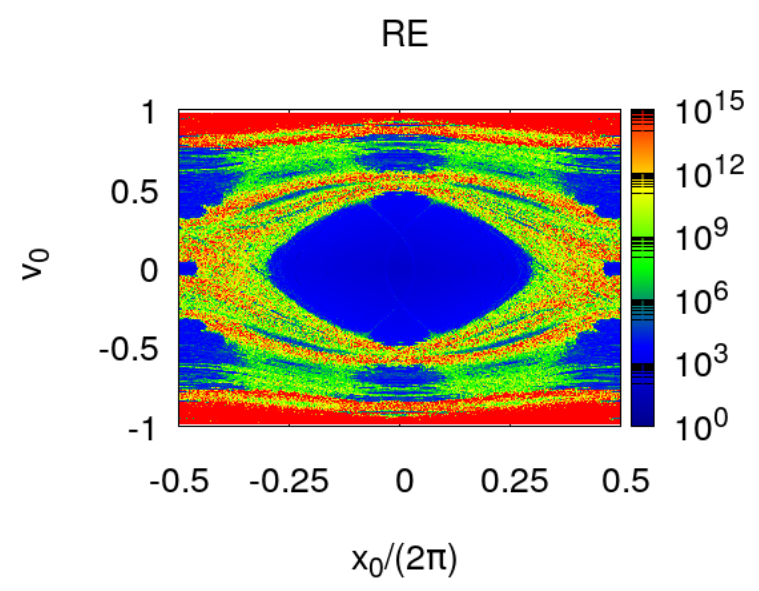

(b)

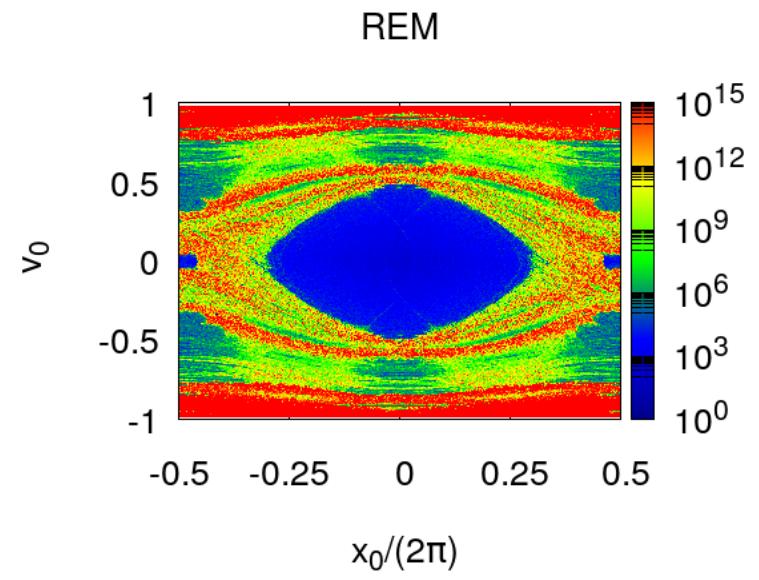

(c)

FIG. 4: From the top: (a) Plot of LE for the 4D reflection map of the 3D waveguide with corrugation amplitude $\varepsilon=0.1$. The initial conditions $\left(x_{0}, y_{0}, v_{x 0}, v_{y 0}\right)$ where $v_{x 0}=v_{0} \cos \phi_{0}$ and $v_{y 0}=v_{0} \sin \phi_{0}$, are chosen in a $2 D$ plane obtained by keeping fixed $y_{0}=\pi / 4$ and $\phi_{0}=0$. The error LE is computed for $N=200$ iterations of the map. We let $\left(x_{0}, v_{0}\right)$ vary on a regular grid of $N_{g} \times N_{g}$ points, with $N_{g}=200$, chosen in the the rectangle $[-\pi, \pi] \times[-1,1]$. (b) The same plot of RE. (c) The same plot of REM. 
LE

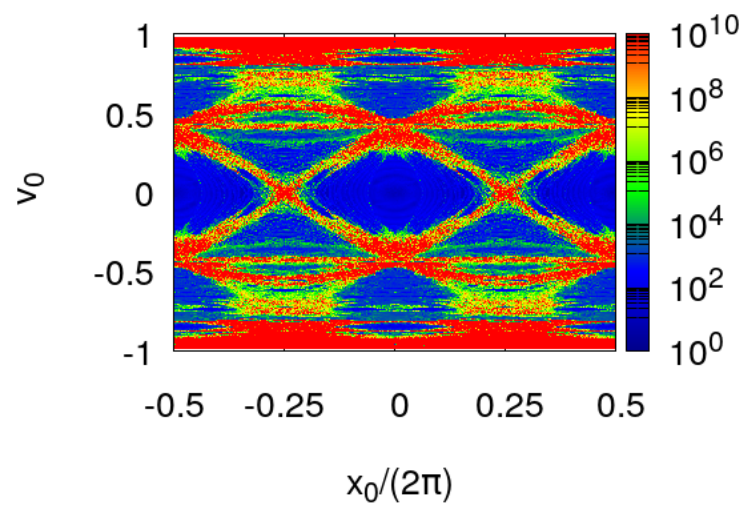

(a)

RE

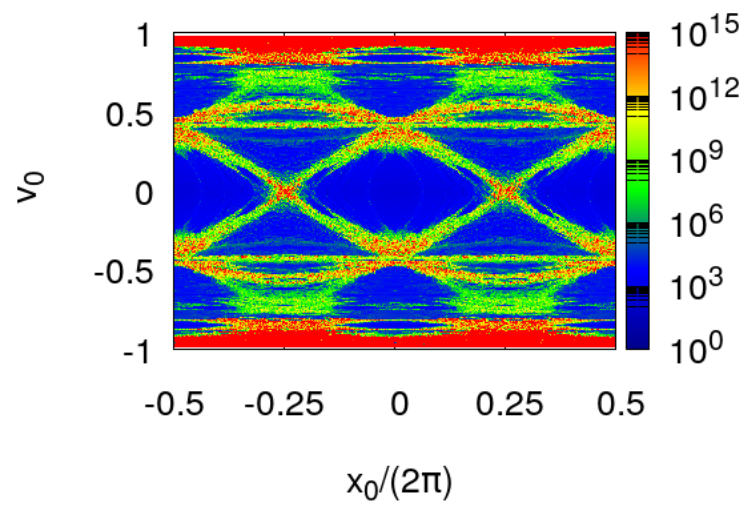

(b)

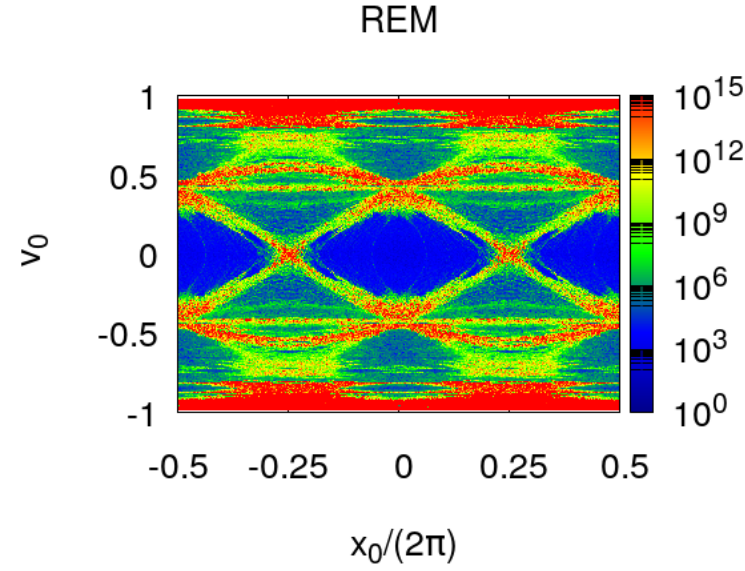

(c)

FIG. 5: Same plots as figure 4 for LE, RE, REM for $\varepsilon=0.1$ and fixed values of $y_{0}=\pi / 2$ and $\phi_{0}=0$. The number of iterations and grid points are the same $N=200, N_{g}=200$.
LE

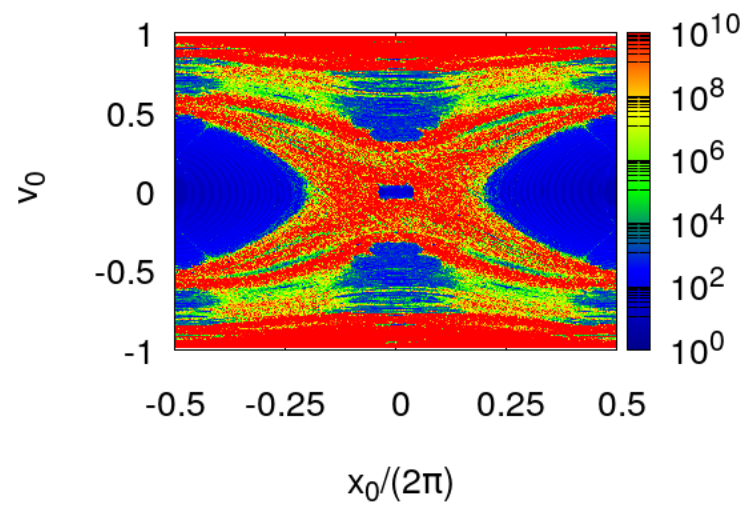

(a)

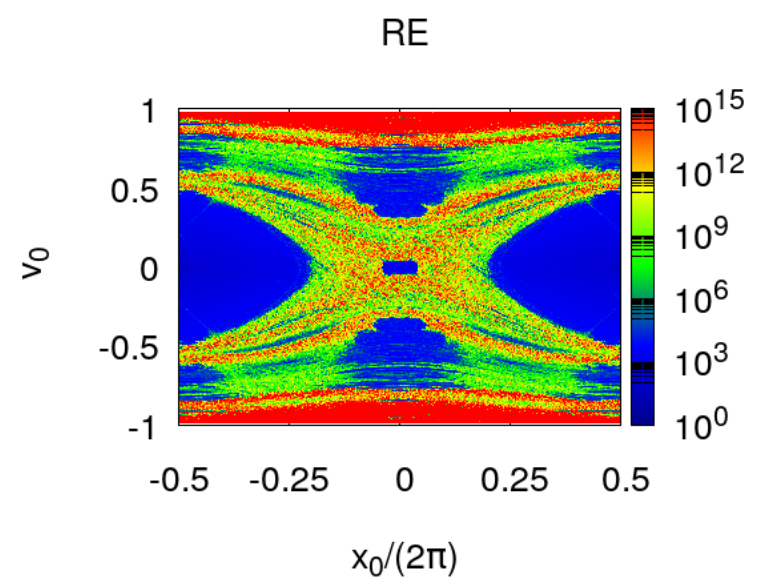

(b)

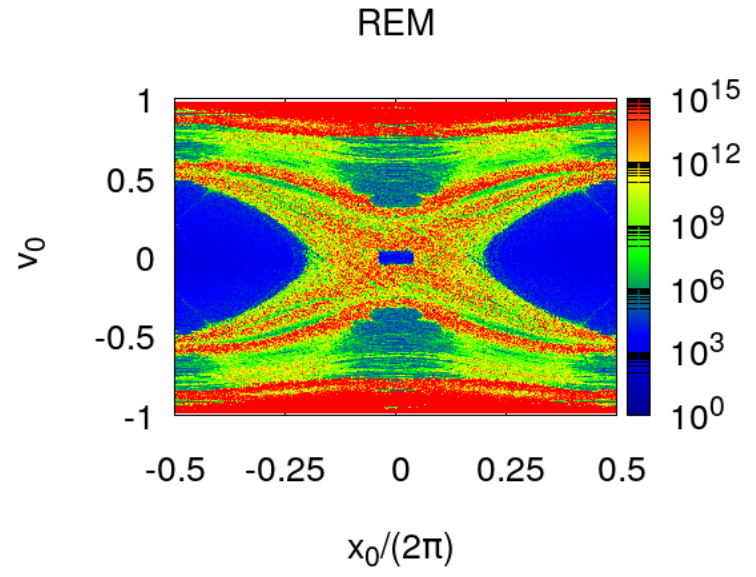

(c)

FIG. 6: Same plots as figure 4 for LE, RE, REM for $\varepsilon=0.1$ and fixed values of $y_{0}=3 \pi / 4$ and $\phi_{0}=0$. The number of iterations and grid points are the same $N=200, N_{g}=200$. 


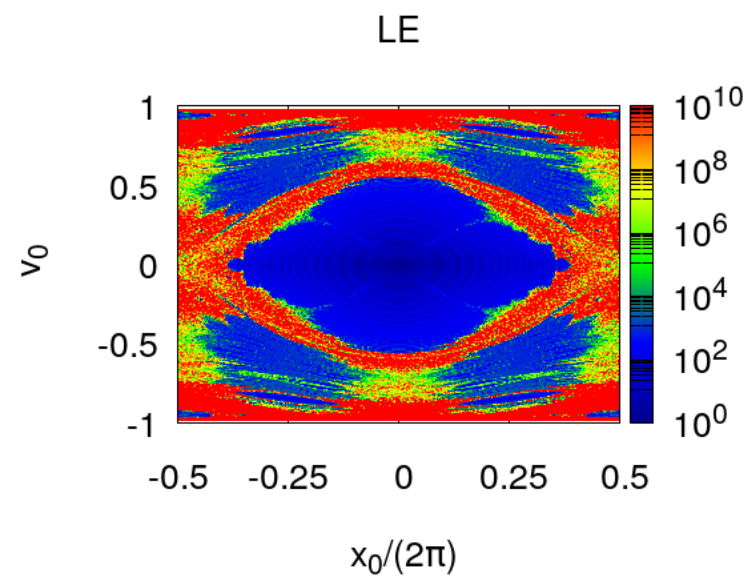

(a)

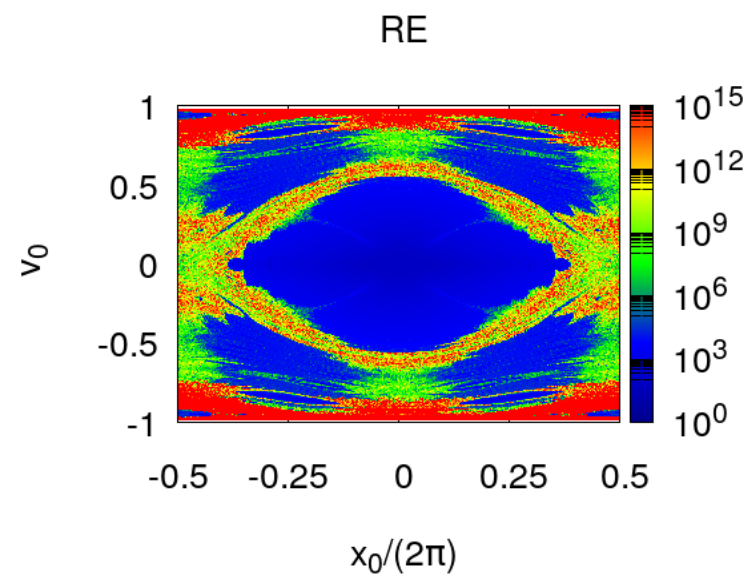

(b)

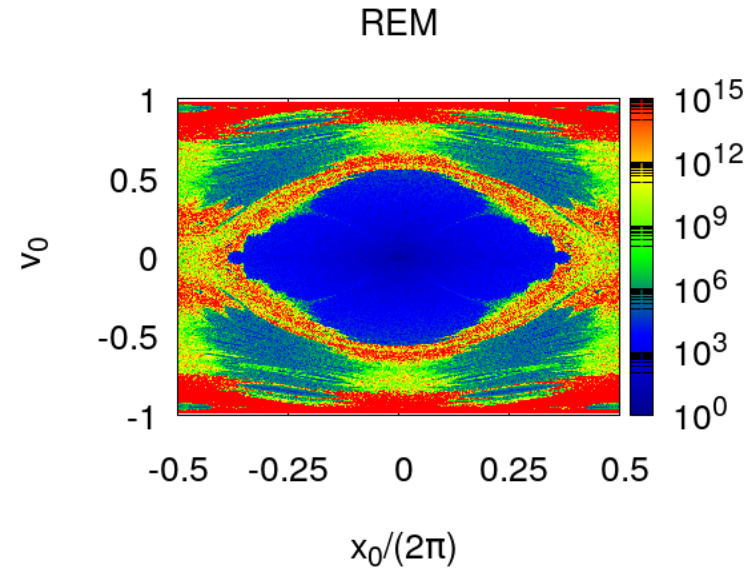

(c)

FIG. 7: Same plots as figure 3 for LE, RE, REM for $\varepsilon=0.1$ and fixed values of $y_{0}=0$ and $\phi_{0}=\pi / 2.01$. The number of iterations and grid points are the same $N=200, N_{g}=200$.
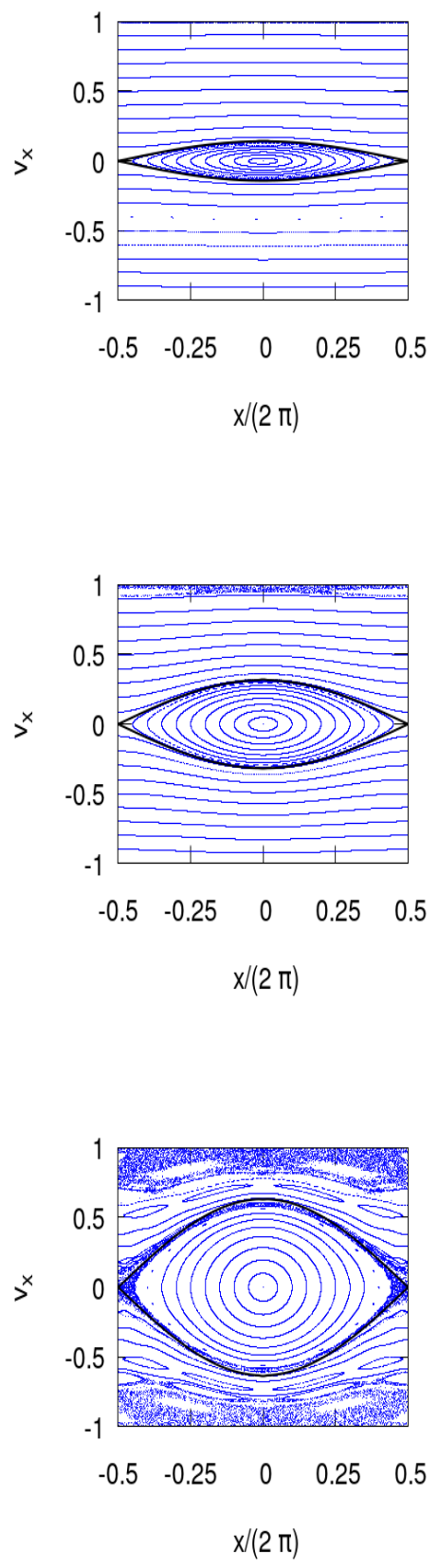

FIG. 8: Phase space portraits of the 2D map with the separatrix, black line, given by equation (40), for different values of the corrugation amplitude. Top frame: $\varepsilon=0.005$.

Center frame: $\varepsilon=0.025$. Bottom frame: $\varepsilon=0.1$. 

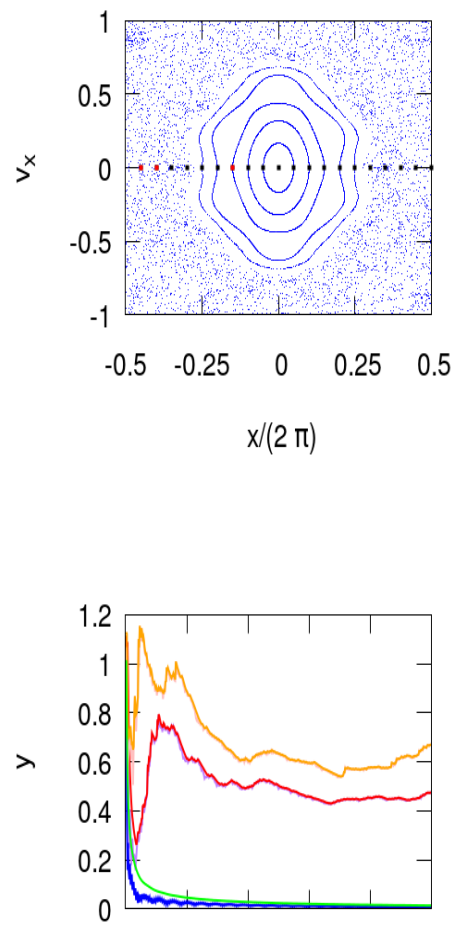

$\begin{array}{llllll}0 & 100 \quad 200 & 300 \quad 400 \quad 500\end{array}$

n

FIG. 9: Top panel: phase portrait of the 2D ray map for $\varepsilon=0.25$. The blue and red dots correspond to the initial conditions. Bottom panel: dependence on $n$ of $C_{n}^{L}$ and $C_{n}^{R}$ for the chaotic orbits whose initial points are the first two red dots in left panel (LE pink, RE orange for the first orbit, LE purple, RE red for the second orbit) and the regular orbit whose initial point is the last red point (LE blue, RE green)

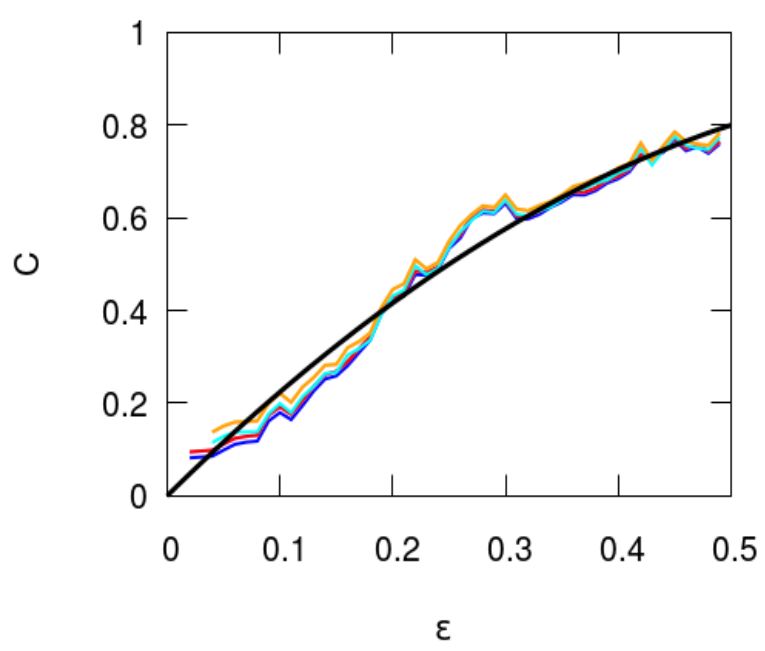

FIG. 10: Growth of the average channel capacities (on a regular grid of 400 points) with $\varepsilon$ for two values of $n$ : $C_{L n}$ cyan for $n=100$, blue for $n=200$ and $C_{R n}$, orange for $n=100$, red for $n=200$. The black line is a quadratic fit given by equation 411 
${ }^{1}$ A. Bazzani, P. Freguglia, and G. Turchetti, Hamiltonian Analytical Optics and Simulations of Betatronic Motion by Optical Devices, in Nonlinear Dynamics and Collective Effects in Particle Beam Physics, World Scientific, pp. 23-46, (2019).

${ }^{2}$ L.A.Bunimovich On the Ergodic Properties of Nowhere Dispersing Billiards, Commun Math Phys. 65 (3), 295-312, (1979).

${ }^{3}$ J. Redmond, S. Tabachnikov Introducing symplectic billiards

https://www.math.psu.edu/tabachni/prints/Notes5.pdf

${ }^{4} \mathrm{~S}$. Tabachnikov billards

https://www.math.psu.edu/tabachni/Books/billiardsbook.pdf

${ }^{5} \mathrm{~S}$. Woo Park An introduction to dynamical billiards

https://math.uchicago.edu/ may/REU2014/REUPapers/Park.pdf

${ }^{6}$ D. Holm and G. Kovacic, Homoclinic chaos for ray optics in a fiber, Physica D 51, p. 177 (1991).

${ }^{7}$ S. S. Abdullaev and G. M. Zavlaskii, Classical nonlinear dynamics and chaos of rays in problems of wave propagation in inhomogeneous media, Usp. Fiz. Nauk. 161, p. 1 (1991).

${ }^{8}$ D. Douglas, Chaotic billiard lasers, Nature 465, p. 696 (2010).

${ }^{9}$ S. Creagh, Directional Emission from Weakly Eccentric Resonators, Phys. Rev. Lett. 98, p. 153901 (2007).

${ }^{10} \mathrm{G}$. Tanner, Dynamical energy analysis Determining wave energy distributions in vibro-acoustical structures in the high-frequency regime, Journal of Sound and Vibration 98, p. 153901 (2007).

${ }^{11}$ E. Leonel, D. da Costa and C. Dettmann, Scaling invariance for the escape of particles from a periodically corrugated waveguide, Physics Letters A 376, 421 (2012).

${ }^{12}$ J. de Oliveira, C. Dettmann, D. , da Costa and E. Leonel, Scaling invariance of the diffusion coefficient in a family of two-dimensional Hamiltonian mappings,Phys. Rev. E 87, p. 062904, (2013).

${ }^{13}$ G. Gradoni, J.-H. Yeh, B. Xiao, T. Antonsen, S. Anlage and O. E., Predicting the statistics of wave transport through chaotic cavities by the random coupling model: A review and recent progress, Wave Motion 51, 606 (2014).

${ }^{14}$ G. Forte, F. Cecconi and A. Vulpiani, Transport and fluctuationdissipation relations in asymptotic and preasymptotic diffusion across channels with variable section, Phys. Rev E 90, p. 062110, (2014). http://denali.phys.uniroma1.it/ cecconif/MyPapersPDF/gforte_PRE90.pdf

${ }^{15}$ F. Cecconi, V. Blakaj, G. Gradoni and A. Vulpiani, Diffusive transport in highly corrugated channels, Phys. Lett. A, 383, pp. 1084-1091, (2018).

${ }^{16}$ C. Froeschlé and E. Lega. On the Structure of Symplectic Mappings. The Fast Lyapunov Indicator: a Very Sensitive Tool. Celestial Mechanics and Dynamical Astronomy, 78, 167-195, 2000.

${ }^{17}$ C. Froeschlé, M. Guzzo and E. Lega. Graphical Evolution of the Arnold Web: From Order to Chaos. Science, 289, 2108-2110, September 2000.

${ }^{18} \mathrm{C}$. Skokos. Alignment indices: a new, simple method for determining the ordered or chaotic nature of orbits. Journal of Physics A Mathematical General, 34, 10029-10043, 2001.

${ }^{19} \mathrm{C}$. Skokos The Lyapunov Characteristic Exponents and Their Computation,J. Souchay and R. Dvorak (eds.), Lecture Notes in Physics, Berlin Springer Verlag Vol. 790, March 2010.

${ }^{20}$ R. Barrio. Theory and Applications of the Orthogonal Fast Lyapunov Indicator (OFLI and OFLI2) Methods. Chaos Detection and Predictability, 915, 55-92, March 2016.

${ }^{21}$ P. M. Cincotta and C. Simó. Simple tools to study global dynamics in non-axisymmetric galactic potentials - I. A\&AS, 147, 205-228, December 2000.

${ }^{22}$ P. M. Cincotta, C. M. Giordano and C. Simó, Phase space structure of multi-dimensional systems by means of the mean exponential growth factor of nearby orbits, Physica D Nonlinear Phenomena 182, 151 (August 2003).

${ }^{23}$ Z. Sandor, B .Erdi, A. Szell, B. Funk. The relative Lyapunov indicator: an efficient method of chaos detection, Celestial Mechanics and Dynamical Astronomy 90, p. 127-138, (2004). https://arxiv.org/pdf/1205.0875.pdf

${ }^{24} \mathrm{Ch}$. Skokos, T. Bountis, Ch. Antonopoulos. Geometrical properties of local dynamics in Hamiltonian systems: The Generalized Alignment Index (GALI) method, Physica D 231, p. 3054, (2007).

${ }^{25} \mathrm{Ch}$. Skokos, T. Manos. The smaller (SALI) and the generalized (GALI) alignment indices: Efficient methods of chaos detection, Chaos Detection and Predictability Springer Lecture Notes in Physics, Editors: Ch. Skokos, Charalampos. G.A. Gottwald, J. Laskar (Eds.). https://arxiv.org/pdf/1412.7401.pdf
${ }^{26} \mathrm{C}$. Voglis, G. Contopoulos. The relative Lyapunov indicator: an efficient method of chaos detection Phys. Rev. E Vol 57, 372-377 (1998)

27. C. Voglis, G. Contopoulos, C. Efthymiopoulos. Detection of ordered and chaotic motion using the dynamical spectra Celestial Mechanics and Dynamical Astronomy Vol 73, 211-220 (1999)

${ }^{28}$ N. P. Maffione, L. A. Darriba - P. M. Cincotta $\cdot$ C. M. Giordano, comparison of different indicators of chaos based on the deviation vectors. Application to symplectic mappings Celestial Mechanics and Dynamical Astronomy 111 (2011) https://arxiv.org/abs/1108.2196

${ }^{29}$ N. P. Maffione, L. A. Darriba - P. M. Cincotta - C. M. Giordano, Comparative study of variational chaos indicators and ODEs numerical integrators, International Journal of Bifurcation and Chaos World Scientific Publishing Company (2012) 22, 1230033 (2012)

${ }^{30} \mathrm{D}$. Ruelle, An inequality for the entropy of differential maps, Bol. Soc. Bras. Mat., 9, 83-87, (1978).

${ }^{31}$ J. Laskar, C. Froeschlé and A. Celletti. The measure of chaos by the numerical analysis of the fundamental frequencies. application to the standard mapping. Physica D: Nonlinear Phenomena, 56, 253-269, 051992.

${ }^{32}$ L. Casetti, C. Clementi, and M. Pettini, Riemannian theory of Hamiltonian chaos and Lyapunov exponents, Phys. Rev. E 54, 5969-5984 (1996).

${ }^{33}$ M. Cerruti-Sola, G. Ciraolo, M. Franzosi, M. Pettini. Riemannian geometry of Hamiltonian chaos: hints for a general theory. Phys. Rev. E Stat. Nonlin. Soft Matter Phys. 78, 046205 (2008)

${ }^{34} \mathrm{G}$. Gottwald, I. Melbourne, Testing for chaos in deterministic systems with noise, Physica D: Nonlinear Phenomena 212, 100-110 (2005)

${ }^{35} \mathrm{~F}$. Panichi, L. Ciotti and G. Turchetti, Fidelity and reversibility in the restricted three body problem, Communications in Nonlinear Science and Numerical Simulation 35, 53 (2015).

${ }^{36} \mathrm{~F}$. Panichi, K. Goździewski and G. Turchetti, The reversibility error method (REM): a new, dynamical fast indicator for planetary dynamics, MNRAS 468, 469 (June 2017).

${ }^{37}$ F. Panichi and G. Turchetti, Fast indicators of orbital stability: a survey on Lyapunov and Reversibility errors INTECH, (2019)

https://www.intechopen.com/online-first/fast-indicators-for-orbitalstability-a-survey-on-lyapunov-and-reversibility-errors

${ }^{38} \mathrm{G}$. Turchetti, F. Panichi and K. Gozdziewski A complete set of fast Lyapunov and Reversibility invariant indicators. To be submitted

${ }^{39}$ D. Faranda, M. F. Mestre and G. Turchetti, Analysis of Round off Errors with Reversibility Test as a Dynamical Indicator, International Journal of Bifurcation and Chaos, 22, p. 1250215 (September 2012).

${ }^{40} \mathrm{G}$. Turchetti, S. Vaienti and F. Zanlungo. Asymptotic distribution of global errors in the numerical computations of dynamical systems. Physica A Statistical Mechanics and its Applications, 389, 4994-5006, (November 2010).

${ }^{41}$ E. Hairer, C. Lubich and G. Wanner. Geometric Numerical Integration: Structure-Preserving Algorithms for Ordinary Differential Equations; 2nd ed. Springer, Dordrecht, (2006).

${ }^{42} \mathrm{G}$. Turchetti, F. Panichi Birkhoff normal forms and stability indicators for betatronic motion Proceedings of the NOCE workshop, Arcidosso sept. 2017, S. Di Mitri, S. Chattopadhyay, M. Cornacchia Editors, World Scientific (2019)

${ }^{43}$ Oseledets, V. I. (1968). A multiplicative ergodic theorem. characteristic Ljapunov, exponents of dynamical systems. Trudy Moskovskogo Matematicheskogo Obshchestva, 19:179-210.

${ }^{44} \mathrm{G}$. Friedland and A. Metere (eds.),Isomorphism between Maximum Lyapunov Exponent and Shannon?s Channel Capacity, https://arxiv.org/pdf/1706.08638.pdf, January 2018.

${ }^{45}$ Tim Holliday, Andrea Goldsmith, Peter W Glynn Capacity of Finite State Channels Based on Lyapunov Exponents of Random Matrices September 2006IEEE Transactions on Information Theory 52(8):3509 - 3532 DOI: 10.1109/TIT.2006.878230 (2006)

${ }^{46}$ Tim Holliday, Peter Glynn, Andrea Goldsmith On Entropy and Lyapunov Exponents for Finite-State Channels http://www.yaroslavvb.com/papers/holliday-entropy.pdf

${ }^{47} \mathrm{~J} . \quad$ M. Ebeid Relating information theoretic limits to the Lyapunov exponents of a dynamical system https://www.ideals.illinois.edu/bitstream/handle/2142/16882/Ebeid_HaniJames.pdf?sequence $=1 \&$ is Allowed $=\mathrm{y}$ 